\title{
Spectral asymptotics of Laplacians related to one-dimensional graph-directed self-similar measures with overlaps
}

\author{
Sze-Man Ngai and Yuanyuan Xie
}

\begin{abstract}
For the class of graph-directed self-similar measures on $\mathbf{R}$, which could have overlaps but are essentially of finite type, we set up a framework for deriving a closed formula for the spectral dimension of the Laplacians defined by these measures. For the class of finitely ramified graph-directed self-similar sets, the spectral dimension of the associated Laplace operators has been obtained by Hambly and Nyberg [6]. The main novelty of our results is that the graphdirected self-similar measures we consider do not need to satisfy the graph open set condition.
\end{abstract}

\section{Introduction}

Let $U \subseteq \mathbf{R}^{d}$ be a bounded domain with smooth boundary, $\Delta$ be the Dirichlet Laplacian on $U,\left\{\lambda_{n}\right\}$ be the eigenvalues of $-\Delta$, and $N(\lambda,-\Delta)$ be the number of eigenvalues that do not exceed $\lambda$. Weyl [24] proved the following asymptotic formula for the Dirichlet Laplacian:

$$
N(\lambda,-\Delta)=\frac{\mathcal{B}_{d}}{(2 \pi)^{d}}|U| \lambda^{d / 2}+o\left(\lambda^{d / 2}\right)=\frac{1}{(4 \pi)^{d / 2} \Gamma(d / 2+1)}|U| \lambda^{d / 2}+o\left(\lambda^{d / 2}\right),
$$

The authors are supported in part by the National Natural Science Foundation of China, grant 11771136, and Construct Program of the Key Discipline in Hunan Province. The first author is also supported in part by the Hunan Province Hundred Talents Program and a Faculty Research Scholarly Pursuit Funding from Georgia Southern University.

Yuanyuan Xie is corresponding author.

Key words and phrases: fractal, spectral dimension, graph-directed self-similar measure, essentially of finite type.

2010 Mathematics Subject Classification: primary 28A80, 35P20; secondary 35J05. 
where $|U|$ denotes the $d$-dimensional volume of $U$ and $\mathcal{B}_{d}$ is the volume of the unit ball in $\mathbf{R}^{d}$. We point out that in our paper [19, Equation (1.1)], the factor $(2 \pi)^{d}$ is incorrectly typed as $(4 \pi)^{d / 2}$.

In this paper, we show that either the $\operatorname{limit} \lim _{\lambda \rightarrow \infty} \frac{N\left(\lambda,-\Delta_{\nu}\right)}{(\ln \lambda)^{m} \lambda^{d_{s} / 2}}$ equals a nonnegative constant or $\frac{N\left(\lambda,-\Delta_{\nu}\right)}{(\ln \lambda)^{m} \lambda^{d_{s} / 2}}$ is asymptotically a nonzero periodic function of $\ln \lambda$, depending on $\nu$, where $m \geq 0,-\Delta_{\nu}$ is a Dirichlet Laplacian on a domain (see definition below), and $d_{s}$ is the spectral dimension. There has been considerable interest in studying the spectral dimension on various fractals or domains supporting a measure. McKean and Ray [15] computed the spectral dimension of the Laplacian defined by the Cantor measure. Fujita [4], Naimark and Solomyak [16] studied the spectral dimension of the Laplace operators defined by self-similar measures satisfying the open set condition (OSC) (see [8]). Kigami and Lapidus [11] obtained the spectral dimension of Laplacians on post-critically finite (p.c.f.) self-similar sets with a harmonic structure. Croydon and Hambly [2] and [5] studied the spectral dimension on the continuum random tree and random recursive affine nested fractals. For finitely ramified graph-directed self-similar sets, Hambly and Nyberg [6] studied the spectral dimension of the associated Laplace operators. Freiberg [3] investigated spectral asymptotics of generalized measure geometric Laplacians on Cantor like sets. Kajino [9] and [10] studied asymptotics of the partition functions associated with self-similar sets. Alonso-Ruiz and Freiberg [1] obtained the spectral dimension of Laplacians on Hanoi attractors.

We also study the relationship between $d_{s}$ and $d_{f}$, where $d_{f}$ is the Hausdorff dimension of the support of $\nu$ with respect to the Euclidean metric. For $d=1$, Solomyak and Verbitsky [21] proved that

$$
d_{s} \leq 2 d_{f} /\left(1+d_{f}\right)
$$

and moreover, equality holds if and only if $p_{i}=\rho_{i}^{d_{f}}$, where $p_{i}$ and $\rho_{i}$ are the probability weights and the contraction ratios of the iterated function system (IFS), respectively.

We say that an IFS or a graph-directed iterated function system (GIFS), as well as any associated self-similar measure or graph-directed self-similar measure, has overlaps, if (OSC) or the graph open set condition (GOSC) (see Section 2.1) fails. In this case, it is much harder to compute the spectral dimension. For a class of IFSs on $\mathbf{R}$ with overlaps and satisfying second-order identities (see [22]), the first author [17] computed the spectral dimension of the Laplacians defined by the corresponding measures. Tang and the authors [19] defined measures that are essentially of finite type (EFT), a property describing the finiteness of basic measure types, and computed the spectral dimension of the Laplacian defined by a self-similar measure satisfying (EFT). The first author and Tang [18] computed 
the spectral dimension of the Laplacians defined by a special class of graph-directed self-similar measures with overlaps. This paper studies the eigenvalue asymptotics of the Dirichlet Laplacians defined by graph-directed self-similar measures with overlaps in much greater generality.

Let $\Omega \subseteq \mathbf{R}^{d}$ be a bounded open set, and $\mu$ be a positive finite Borel measure on $\mathbf{R}^{d}$ with $\operatorname{supp}(\mu) \subseteq \bar{\Omega}$ and $\mu(\Omega)>0$. We assume that the Poincaré inequality $(\mathrm{PI})$ for $\mu$ holds: There exists a constant $C>0$ such that

$$
\int_{\Omega}|u|^{2} d \mu \leq C \int_{\Omega}|\nabla u|^{2} d x, \quad \text { for all } u \in C_{c}^{\infty}(\Omega)
$$

(see, e.g., [7], [14] and [16]). (PI) implies that each equivalence class $u \in H_{0}^{1}(\Omega)$ contains a unique (in the $L^{2}(\Omega, \mu)$ sense) member $\bar{u}$ that belongs to $L^{2}(\Omega, \mu)$ and satisfies both conditions below:

(1) there exists a sequence $\left\{u_{n}\right\}$ in $C_{c}^{\infty}(\Omega)$ such that $u_{n} \rightarrow \bar{u}$ in $H_{0}^{1}(\Omega)$ and $u_{n} \rightarrow \bar{u}$ in $L^{2}(\Omega, \mu)$

(2) $\bar{u}$ satisfies (1.3).

We call $\bar{u}$ the $L^{2}(\Omega, \mu)$-representative of $u$. Define a mapping $\iota: H_{0}^{1}(\Omega) \rightarrow$ $L^{2}(\Omega, \mu)$ by $\iota(u)=\bar{u}$. It is easy to see that $\iota$ is a bounded linear operator, but not necessarily injective. Consider the subspace $\mathcal{N}$ of $H_{0}^{1}(\Omega)$ defined as

$$
\mathcal{N}:=\left\{u \in H_{0}^{1}(\Omega):\|\iota(u)\|_{L^{2}(\Omega, \mu)}=0\right\} .
$$

It follows from the continuity of $\iota$ that $\mathcal{N}$ is a closed subspace of $H_{0}^{1}(\Omega)$. Let $\mathcal{N}^{\perp}$ be the orthogonal complement of $\mathcal{N}$ in $H_{0}^{1}(\Omega)$. Then $\iota: \mathcal{N}^{\perp} \rightarrow L^{2}(\Omega, \mu)$ is injective. With a slight abuse of notation, we will denote $\bar{u}$ by $u$.

Consider a non-negative bilinear form $\mathcal{E}(\cdot, \cdot)$ in $L^{2}(\Omega, \mu)$ given by

$$
\mathcal{E}(u, v):=\int_{\Omega} \nabla u \cdot \nabla v d x
$$

with domain $\operatorname{dom} \mathcal{E}=\mathcal{N}^{\perp}$. (PI) implies that $(\mathcal{E}, \operatorname{dom} \mathcal{E})$ is a closed quadratic form on $L^{2}(\Omega, \mu)$. Hence these exists a non-negative self-adjoint operator on $L^{2}(\Omega, \mu)$, which we denote by $-\Delta_{\mu}$ and call the (Dirichlet) Laplacian with respect to $\mu$, such that $\operatorname{dom} \mathcal{E}=\operatorname{dom}\left(-\Delta_{\mu}\right)^{1 / 2}$ and $\mathcal{E}(u, v)=\left\langle\left(-\Delta_{\mu}\right)^{1 / 2} u,\left(-\Delta_{\mu}\right)^{1 / 2} v\right\rangle_{L^{2}(\Omega, \mu)}$ for all $u, v \in \operatorname{dom} \mathcal{E}$. Let $u \in \operatorname{dom} \mathcal{E}$. Then $u \in \operatorname{dom} \Delta_{\mu}$ holds if and only if there exists $f \in$ $L^{2}(\Omega, \mu)$ such that $\mathcal{E}(u, v)=\langle f, v\rangle_{L^{2}(\Omega, \mu)}$ for all $v \in \operatorname{dom} \mathcal{E}$, where $-\Delta_{\mu} u=f$. We remark that if $d=1$, then (PI) holds for any such $\mu$, and thus $-\Delta_{\mu}$ is well defined.

We assume that $L^{2}(\Omega, \mu)$ is infinite dimensional. It is known (see, e.g., [7]) that there exists an orthonormal basis $\left\{\varphi_{n}\right\}_{n=1}^{\infty}$ of $L^{2}(\Omega, \mu)$ consisting of the eigenfunctions of $-\Delta_{\mu}$. The eigenvalues $\lambda_{n}=\lambda_{n}\left(-\Delta_{\mu}\right)$ satisfy $0<\lambda_{1} \leq \lambda_{2} \leq \ldots$ and $\lim _{n \rightarrow \infty} \lambda_{n}=\infty$. 
Let $N\left(\lambda,-\Delta_{\mu}\right)$ be the number of eigenvalues of $-\Delta_{\mu}$ (counting multiplicity) which do not exceed $\lambda$, i.e.,

$$
N\left(\lambda,-\Delta_{\mu}\right):=\#\left\{n: \lambda_{n} \leq \lambda\right\},
$$

where \#A denotes the cardinality of a set $A$. Define the lower and upper spectral dimensions of $-\Delta_{\mu}$, respectively, as

$$
\underline{d}_{s}\left(-\Delta_{\mu}\right):=\varliminf_{\lambda \rightarrow \infty} \frac{2 \ln N\left(\lambda,-\Delta_{\mu}\right)}{\ln \lambda} \quad \text { and } \quad \bar{d}_{s}\left(-\Delta_{\mu}\right):=\varlimsup_{\lambda \rightarrow \infty} \frac{2 \ln N\left(\lambda,-\Delta_{\mu}\right)}{\ln \lambda} .
$$

If $\underline{d}_{s}\left(-\Delta_{\mu}\right)=\bar{d}_{s}\left(-\Delta_{\mu}\right)$, the common value, denoted $d_{s}\left(-\Delta_{\mu}\right)$ (or simply $d_{s}$ if no confusion is possible), is called the spectral dimension of $-\Delta_{\mu}$; it measures the asymptotic growth rate of the eigenvalue counting function as well as the magnitude of the $n$-th eigenvalue.

(EFT) is introduced in [19]. Let $\mu=\sum_{i=1}^{q} \mu_{i}$ be the graph-directed self-similar measure defined by a GIFS $G=(V, E)$ on $\mathbf{R}^{d}$, where $V=\{1, \ldots, q\}$ is the set of vertices and $E$ is the set of directed edges with each edge beginning and ending at a vertex. We say that $\mu$ satisfies (EFT) (see Definition 2.1) if there exist a family of bounded open subsets $\left\{\Omega_{i}\right\}_{i=1}^{q}$ with $\Omega_{i} \subseteq \mathbf{R}^{d}, \operatorname{supp}\left(\mu_{i}\right) \subseteq \bar{\Omega}_{i}$, and $\mu\left(\Omega_{i}\right)>0$, and a finite family $\mathbf{B}:=\left\{B_{1, \ell}: \ell \in \Gamma\right\}$ of measure disjoint cells (i.e., subsets of $\bigcup_{i=1}^{q} \Omega_{i}$ with positive $\mu$-measure), $B_{1, \ell} \subseteq \Omega_{i_{\ell}}$ for some $i_{\ell} \in V$, such that for any $\ell \in \Gamma$, there is a family of $\mu$-partitions $\left\{\mathbf{P}_{k, \ell}\right\}_{k \geq 1}$ of $B_{1, \ell}$ satisfying the following conditions: (1) $\mathbf{P}_{1, \ell}=\left\{B_{1, \ell}\right\}$, and there exists some $B \in \mathbf{P}_{2, \ell}^{1}$ (see $\left.(2.6)\right)$ such that $B \neq B_{1, \ell}$, where $\mathbf{P}_{2, \ell}^{1}$ is the collection of all sets $B$ in $\mathbf{P}_{2, \ell}$ that are $\mu$-equivalent to some $B_{1, \ell}$ for $\ell \in \Gamma$; (2) for any $k \geq 2, \mathbf{P}_{k+1, \ell}^{1}$ contains all cells in $\mathbf{P}_{k, \ell}^{1}$ that are $\mu$-equivalent to some cell in $\mathbf{B}$; (3) the sum of the $\mu$-measures of those cells $B \in \mathbf{P}_{k, \ell}$ that are not $\mu$-equivalent to any cell in $\mathbf{B}$ tends to 0 as $k \rightarrow \infty$. In this case, we call $\left\{\Omega_{i}\right\}_{i=1}^{q}$ an EFT-family, B a basic family of cells, and $(\mathbf{B}, \mathbf{P}):=\left(\left\{B_{1, \ell}\right\},\left\{\mathbf{P}_{k, \ell}\right\}_{k \geq 1}\right)_{\ell \in \Gamma}$ a basic pair. We say that $(\mathbf{B}, \mathbf{P})$ is regular if each cell $B \in \bigcup_{k \geq 1, \ell \in \Gamma} \mathbf{P}_{k, \ell}$ is connected, and for any $\ell \in \Gamma$, there exist some similitude $\tau_{\ell}$, some $\Omega_{j_{\ell}}$, and some constant $w(\ell)>0$ such that $\tau_{\ell}\left(\Omega_{j_{\ell}}\right) \subseteq B_{1, \ell}$ and $\mu \geq w(\ell) \mu \circ \tau_{\ell}^{-1}$ on $\tau_{\ell}\left(\Omega_{j_{\ell}}\right)$.

Let $\mu=\sum_{i=1}^{q} \mu_{i}$ be the graph-directed self-similar measure defined by a GIFS $G=(V, E)$ on R. Assume that $G$ has $\eta=\eta(G)$ strongly connected components, and $\mu$ satisfies (EFT) with $\left\{\Omega_{i}\right\}_{i=1}^{q}$ being an EFT-family and assume that there exists a regular basic pair $(\mathbf{B}, \mathbf{P}):=\left(\left\{B_{1, \ell}\right\},\left\{\mathbf{P}_{k, \ell}\right\}_{k \geq 1}\right)_{\ell \in \Gamma}$. For $m=1, \ldots, \eta$, let

(1.6) $\mathcal{S C}_{m}:=\{i \in V: i$ is contained in the $m$-th strongly connected component $\}$,

and for $i \in \mathcal{S C}_{m}$, let

$$
\Gamma_{i}:=\left\{\ell \in \Gamma: B_{1, \ell} \subseteq \Omega_{i}\right\}
$$


Under these assumptions, we can derive renewal equations for the eigenvalue counting functions, and express them in vector form as:

$$
\mathbf{f}=\mathbf{f} * \mathbf{M}_{\boldsymbol{\alpha}}+\mathbf{z}
$$

where

$$
\begin{aligned}
& \boldsymbol{\alpha}=\left(\alpha_{1}, \ldots, \alpha_{\eta}\right), \quad \alpha_{m} \in \mathbf{R} \text { for } m=1, \ldots, \eta ; \\
& \mathbf{f}=\mathbf{f}^{(\boldsymbol{\alpha})}(t)=\left[f_{\ell}^{\left(\alpha_{m}\right)}(t)\right]_{\ell \in \Gamma}, \quad t \in \mathbf{R} ; \\
& \mathbf{M}_{\boldsymbol{\alpha}}=\left[\mu_{\ell \ell^{\prime}}^{\left(\alpha_{m}\right)}\right]_{\ell, \ell^{\prime} \in \Gamma} \quad \text { is a finite matrix of Borel measures on } \mathbf{R} ; \\
& \mathbf{z}=\mathbf{z}^{(\alpha)}(t)=\left[z_{\ell}^{\left(\alpha_{m}\right)}(t)\right]_{\ell \in \Gamma} \text { is a vector of error functions. }
\end{aligned}
$$

Let

$$
\mathbf{M}(\boldsymbol{\alpha} ; \infty):=\left[\mu_{\ell \ell^{\prime}}^{\left(\alpha_{m}\right)}(\mathbf{R})\right]_{\ell, \ell^{\prime} \in \Gamma}
$$

For each $\ell \in \Gamma_{i}$ and $\alpha_{m} \geq 0$, define

$$
F_{\ell}\left(\alpha_{m}\right):=\sum_{\ell^{\prime} \in \Gamma} \mu_{\ell \ell^{\prime}}^{\left(\alpha_{m}\right)}(\mathbf{R}), \quad D_{\ell}:=\left\{\alpha_{m} \geq 0: F_{\ell}\left(\alpha_{m}\right)<\infty\right\}, \quad \widetilde{\alpha}_{\ell}:=\inf D_{\ell}
$$

If the error functions decay exponentially to 0 as $t \rightarrow \infty$, then $d_{s}\left(-\Delta_{\mu}\right)$ is given by the maximum of the unique set of non-negative real numbers $\alpha_{1}, \ldots, \alpha_{\eta}$ such that spectral radii of $\mathbf{M}(\boldsymbol{\alpha} ; \infty)$ and all of the classes (see definition below) are equal to 1 , where $\boldsymbol{\alpha}=\left(\alpha_{1}, \ldots, \alpha_{\eta}\right)$.

We denote the convolution of a function $a$ with a measure $b$ by

$$
b * a(x)=a * b(x)=\int_{0}^{t} a(x-s) b(d s)
$$

if both $a$ and $b$ are measures, we convolve the distribution function of $a$ with the measure $b$. For two matrices $A, B$ of measures, we write the $i j$-th element of $C(x)=$ $A * B(x)$ as $c_{i j}(x)=\sum_{k} a_{i k} * b_{k j}(x)$.

We call $\gamma=\left(\ell_{1}, \ldots, \ell_{n}\right)$, where $n \geq 2$ and $\ell_{j} \in \Gamma$ for $j=1, \ldots, n$, a path (or $\gamma$-path) from $\ell_{1}$ to $\ell_{n}$. Such a $\gamma$ is called a cycle if $\ell_{1}=\ell_{n}$, and a simple cycle if it is a cycle and all $\ell_{1}, \ldots, \ell_{n-1}$ are distinct. For any path $\gamma=\left(\ell_{1}, \ldots, \ell_{n}\right)$, let $\ell_{j} \in \Gamma_{i}$ and $i \in \mathcal{S C}_{m_{j}}$ for $i \in V, j=1, \ldots, n$, and $m_{j} \in\{1, \ldots, \eta\}$. Define

$$
\mu_{\gamma}=\mu_{\ell_{1} \ell_{2}}^{\left(\alpha_{m_{1}}\right)} * \mu_{\ell_{2} \ell_{3}}^{\left(\alpha_{m_{2}}\right)} * \ldots * \mu_{\ell_{n-1} \ell_{n}}^{\left(\alpha_{m_{n-1}}\right)} .
$$

For $\ell, \ell^{\prime} \in \Gamma$, let $\mathbf{M}_{\ell \ell^{\prime}}^{\boldsymbol{\alpha}}$ be the submatrix of $\mathbf{M}_{\boldsymbol{\alpha}}$ obtained by deleting the $\ell$-th row and $\ell^{\prime}$-th column of $\mathbf{M}_{\boldsymbol{\alpha}}, \mu_{\hat{\ell} \ell^{\prime}}^{(\alpha)}$ be the $\ell^{\prime}$-th column of the matrix $\mathbf{M}_{\boldsymbol{\alpha}}$ with the $\ell$-th 
element removed, and $\mu_{\ell \widehat{\ell}^{\prime}}^{(\alpha)}$ for the $\ell$-th row of $\mathbf{M}_{\boldsymbol{\alpha}}$ with the $\ell^{\prime}$-th element removed. Let

$$
\nu_{\ell}=\mu_{\ell \ell}^{(\alpha)}+\mu_{\ell \hat{\ell}}^{(\alpha)} * \sum_{n=0}^{\infty}\left(\mathbf{M}_{\ell \ell}^{\alpha}\right)^{* n} * \mu_{\hat{\ell} \ell}^{(\alpha)} \quad \text { for } \ell \in \Gamma .
$$

It is known that if $\mathbf{M}(\boldsymbol{\alpha} ; \infty)$ has maximal eigenvalue 1 and is irreducible, then $\nu_{\ell}$ is a probability measure with support given by $\cup\left\{\operatorname{supp}\left(\mu_{\gamma}\right): \gamma\right.$ is a simple cycle in $\left.G\right\}$ (see [12, Lemma 2.3.]). If $\operatorname{supp}\left(\nu_{\ell}\right)$ is contained in a discrete subgroup of $\mathbf{R}$, we call $\nu_{\ell}$ lattice; otherwise we call it non-lattice. By the irreducibility, we see that if $\nu_{1}$ is non-lattice, then $\nu_{\ell}$ is non-lattice for all $\ell$.

For $\ell, \ell^{\prime} \in \Gamma$, we say that $\ell$ has access to $\ell^{\prime}$ (or $\ell^{\prime}$ has access from $\ell$ ) if there is a path from $\ell$ to $\ell^{\prime}$. $\ell$ and $\ell^{\prime}$ are said to communicate if they have access to each other. Using the communication relation, we can partition all $\ell \in \Gamma$ into equivalence classes. The spectral radius of a class is the spectral radius of the matrix obtained by restricting $\mathbf{M}(\boldsymbol{\alpha} ; \infty)$ to that class. A class is called basic if its spectral radius is the same as that of $\mathbf{M}(\boldsymbol{\alpha} ; \infty)$. If a class is not basic, then it is called non-basic. A class $J$ is final if $J$ does not have access to other classes. A chain of classes is a collection of classes such that each class has access to or from another in the collection. The length of a chain is the number of basic classes that it contains. The height of a basic class $C$ is the length of the longest chain of classes which have access to $C$. Let $\mathcal{S}_{m}$ denote the union of basic classes of height $m+1$ for $m \geq 0$.

Theorem 1.1. Let $\mu=\sum_{i=1}^{q} \mu_{i}$ be a graph-directed self-similar measure defined by a strongly connected $G I F S G=(V, E)$ on $\mathbf{R}$. Assume that $\mu$ satisfies $(E F T)$ with $\left\{\Omega_{i}\right\}_{i=1}^{q}$ being an EFT-family and assume that there exists a regular basic pair. Let $\Omega=\bigcup_{i=1}^{q} \Omega_{i}, \Delta_{\mu}$ be the Dirichlet Laplacian defined by $\mu$, and let $\mathbf{M}(\boldsymbol{\alpha} ; \infty), F_{\ell}\left(\alpha_{m}\right)$, $\tilde{\alpha}_{\ell}$ and $\nu_{\ell}$ be defined as in (1.9), (1.10) and (1.11). Assume that for $\eta=1, i \in \mathcal{S C}$, and $\ell \in \Gamma_{i}$, we have $\lim _{\alpha \rightarrow \infty} F_{\ell}(\alpha)=0$ and $\lim _{\alpha \rightarrow \tilde{\alpha}_{\ell}^{+}} F_{\ell}(\alpha)>1$.

(a) There exists a unique $\alpha>0$ such that the spectral radius of $\mathbf{M}(\boldsymbol{\alpha} ; \infty)$ equals 1 , where $\boldsymbol{\alpha}:=(\alpha)$ consists of only one component.

(b) If we assume, in addition, that for the unique $\alpha$ in $(a)$, there exists $\sigma>0$ such that for all $\ell \in \Gamma, z_{\ell}^{(\alpha)}(t)=o\left(e^{-\sigma t}\right)$ as $t \rightarrow \infty$, then $d_{s}=2 \alpha$.

(c) Let $i \in V$ and $\ell \in \Gamma_{i}$ (see (4.1)). If $\nu_{1}$ is non-lattice, then there exists a nonnegative constant $c_{\ell}$ such that

$$
\lim _{t \rightarrow \infty} e^{-\alpha t} N\left(e^{t},-\Delta_{\left.\mu_{i}\right|_{B_{1, \ell}}}\right)=c_{\ell} ;
$$

if $\nu_{1}$ is lattice, then there exists a periodic function $q_{\ell}$ such that

$$
\lim _{t \rightarrow \infty}\left(e^{-\alpha t} N\left(e^{t},-\Delta_{\left.\mu_{i}\right|_{B_{1, \ell}}}\right)-q_{\ell}(t)\right)=0 .
$$


$\underline{0}$

$\underline{S_{e_{1}}\left(\Omega_{1}\right)} S_{e_{2}}\left(\Omega_{2}\right) \quad \underline{S_{e_{3}}\left(\Omega_{1}\right)}$

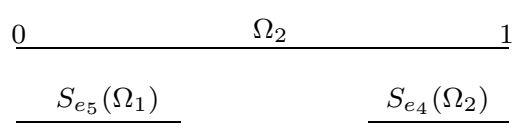

Figure 1. The first iteration of the GIFS defined in (1.12), where $\Omega_{1}=\Omega_{2}=(0,1)$. The figure is drawn with $\rho=1 / 3$ and $r=2 / 7$.

In Section 5 , we illustrate Theorem 1.1 by the following example.

Example 1.2. Let $G=(V, E)$ be a strongly connected GIFS with $V=\{1,2\}$ and $E=\left\{e_{i}: 1 \leq i \leq 5\right\}$, where $e_{1}, e_{3} \in E^{1,1}, e_{2} \in E^{1,2}, e_{4} \in E^{2,2}, e_{5} \in E^{2,1}$. The five similitudes are defined by

$$
\begin{array}{lll}
S_{e_{1}}(x)=\rho x, & S_{e_{2}}(x)=r x+\rho(1-r), & S_{e_{3}}(x)=r x+(1-r), \\
S_{e_{4}}(x)=r x+(1-r), & S_{e_{5}}(x)=\rho x, &
\end{array}
$$

where

$$
\rho+2 r-\rho r \leq 1,
$$

i.e., $S_{e_{2}}(1) \leq S_{e_{3}}(0)$ (see Figure 1).

Corollary 1.3. Let $\mu=\mu_{1}+\mu_{2}$ be a graph-directed self-similar measure defined by a GIFS $G=(V, E)$ in Example 1.2 together with a probability matrix $\left(p_{e}\right)_{e \in E}$. If $\left(p_{e_{1} e_{3}}+p_{e_{2} e_{5}}\right) p_{e_{5}}^{-1} r<1$, then there exists a unique $\alpha>0$ satisfying

$$
\left[1-\left(p_{e_{4}} r\right)^{\alpha}\right]\left[\left(1-\left(p_{e_{1}} \rho\right)^{\alpha}\right)\left(1-\left(p_{e_{3}} r\right)^{\alpha}\right)-\left(\left(p_{e_{1} e_{3}}+p_{e_{2} e_{5}}\right) \rho r\right)^{\alpha}\right]=\left(p_{e_{2} e_{4} e_{5}} \rho r^{2}\right)^{\alpha} .
$$

Moreover, $d_{s}=2 \alpha$. If $\nu_{1}$ is non-lattice, then there exists a non-negative constant $c_{\ell}$ such that

$$
\lim _{t \rightarrow \infty} e^{-\alpha t} N\left(e^{t},-\Delta_{\left.\mu_{i}\right|_{B_{1, \ell}}}\right)=c_{\ell}, \quad \text { for } \ell \in \Gamma_{i}, \ell=1,3,4 \text {, and } i=1,2 ;
$$

if $\nu_{1}$ is lattice, then there exists a periodic function $q_{\ell}$ such that

$$
\lim _{t \rightarrow \infty}\left(e^{-\alpha t} N\left(e^{t},-\Delta_{\left.\mu_{i}\right|_{B_{1, \ell}}}\right)-q_{\ell}(t)\right)=0, \quad \text { for } \ell \in \Gamma_{i}, \ell=1,3,4 \text {, and } i=1,2 .
$$

Remark 1.4. Let $G=(V, E)$ be defined as in Example 1.2, and let $\rho=1 / 3, r=$ $2 / 7, p_{e_{i}}=1 / 4$, and $p_{e_{j}}=1 / 2$ for $i=1,2$ and $j=3,4,5$. The following hold:

(a) numerical approximations yield $d_{s}=0.818596 \ldots$;

(b) the Hausdorff dimension of the associated graph self-similar set is $d_{f}=$ $0.710396 \ldots$, and moreover, $d_{s}<2 d_{f} /\left(1+d_{f}\right)$; 
(c) there exists a non-negative constant $c_{\ell}$ such that

$$
\lim _{t \rightarrow \infty} e^{-\alpha t} N\left(e^{t},-\Delta_{\left.\mu_{i}\right|_{B_{1, \ell}}}\right)=c_{\ell}, \text { for } \ell \in \Gamma_{i}, \ell=1,3,4 \text {, and } i=1,2 .
$$

Remark 1.5. We remark that in Theorem 1.1, it is necessary to assume that $\lim _{\alpha_{m} \rightarrow \infty} F_{\ell}\left(\alpha_{m}\right)=0$ for each $\ell \in \Gamma$. In fact, if we let $\left(p_{e_{1} e_{3}}+p_{e_{2} e_{5}}\right) p_{e_{5}}^{-1} r>1$ in Corollary 1.3 , then $\lim _{\alpha_{m} \rightarrow \infty} F_{\ell}\left(\alpha_{m}\right)=\infty$ for some $m \in\{1, \ldots, \eta\}$ and some $\ell \in \Gamma_{i}$, where $i \in \mathcal{S C}_{m}$. Hence Theorem 1.1(a) does not hold.

Theorem 1.6. Let $\mu=\sum_{i=1}^{q} \mu_{i}$ be a graph-directed self-similar measure on $\mathbf{R}$ defined by a GIFS $G=(V, E)$ that is not strongly connected. Assume that $G$ has $\eta$ strongly connected components. For $m=1, \ldots, \eta$, let $2 \alpha_{m}$ be the spectral dimension of the graph-directed self-similar measure corresponding to the $m$-th strongly connected component. Assume that $\mu$ satisfies (EFT) with $\left\{\Omega_{i}\right\}_{i=1}^{q}$ being an EFT-family and assume that a regular basic pair exists. Let $\Omega=\bigcup_{i=1}^{q} \Omega_{i}$ and $\Delta_{\mu}$ be the Dirichlet Laplacian defined by $\mu$. Let $\mathbf{M}(\boldsymbol{\alpha} ; \infty), F_{\ell}\left(\alpha_{m}\right)$ and $\tilde{\alpha}_{\ell}$ be defined as in (1.9) and (1.10). Assume that for $m=1, \ldots, \eta, i \in \mathcal{S C}_{m}$, and $\ell \in \Gamma_{i}$, we have $\lim _{\alpha_{m} \rightarrow \infty} F_{\ell}\left(\alpha_{m}\right)=0$ and $\lim _{\alpha_{m} \rightarrow \tilde{\alpha}_{\ell}^{+}} F_{\ell}\left(\alpha_{m}\right)>1$.

(a) There exists a unique set of real numbers $\alpha_{1}, \ldots, \alpha_{\eta}$ such that the spectral radii of $\mathbf{M}(\boldsymbol{\alpha} ; \infty)$ and all the other classes equal 1 , where $\boldsymbol{\alpha}:=\left(\alpha_{1}, \ldots, \alpha_{\eta}\right)$.

(b) If we assume, in addition, that for the unique set $\left\{\alpha_{1}, \ldots, \alpha_{\eta}\right\}$ in $(a)$, there exists $\sigma>0$ such that for all $\ell \in \Gamma, z_{\ell}^{(\alpha)}(t)=o\left(e^{-\sigma t}\right)$ as $t \rightarrow \infty$, then we have $d_{s}=2 \alpha$, where $\alpha:=\max \left\{\alpha_{1}, \ldots, \alpha_{\eta}\right\}$.

(c) Let $i \in V$ and $\ell \in \Gamma_{i}$.

(1) If $\ell \in \mathcal{S}_{0}$, then

$$
\lim _{t \rightarrow \infty}\left(e^{-\alpha t} N\left(e^{t},-\Delta_{\left.\mu_{i}\right|_{B_{1, \ell}}}\right)-q_{\ell}(t)\right)=0,
$$

where $q_{\ell}$ is either periodic or non-negative constant depending on whether $\nu_{\ell}$ is lattice or not.

(2) If $m>0$ and $\ell \in \mathcal{S}_{m}$, then there exists a constant $c_{\ell} \geq 0$ such that

$$
\lim _{t \rightarrow \infty} t^{-m} e^{-\alpha t} N\left(e^{t},-\Delta_{\left.\mu_{i}\right|_{B_{1, \ell}}}\right)=c_{\ell} .
$$

(3) If $\ell \notin \mathcal{S}=\bigcup_{m \geq 0} \mathcal{S}_{m}$ and there is no path from $\mathcal{S}$ to $\ell$, then

$$
\lim _{t \rightarrow \infty} e^{-\alpha t} N\left(e^{t},-\Delta_{\left.\mu_{i}\right|_{B_{1, \ell}}}\right)=0 .
$$

(4) If $\ell \notin \mathcal{S}$ and there is a path from $\mathcal{S}_{0}$ to $\ell$, but no path to $\ell$ from $\mathcal{S}_{k}$ for any $k>0$, then

$$
\lim _{t \rightarrow \infty}\left(e^{-\alpha t} N\left(e^{t},-\Delta_{\left.\mu_{i}\right|_{B_{1, \ell}}}\right)-\tilde{q}_{\ell}(t)\right)=0,
$$

for some $\tilde{q}_{\ell}$ which is either non-negative constant or periodic. 
(5) If $\ell \notin \mathcal{S}$ and there is a path from $\mathcal{S}_{m}$ to $\ell$, but no path from $\mathcal{S}_{k}$, for any $k>m>0$, then there is a constant $\tilde{c}_{\ell} \geq 0$ such that

$$
\lim _{t \rightarrow \infty} t^{-m} e^{-\alpha t} N\left(e^{t},-\Delta_{\left.\mu_{i}\right|_{B_{1}, \ell}}\right)=\tilde{c}_{\ell} .
$$

In Subsections 6.1-6.2, we illustrate Theorem 1.6 by the following example.

Example 1.7. Let $G=(V, E)$ be a GIFS that is not strongly connected with $V=\{1,2\}$ and $E=\left\{e_{i}: 1 \leq i \leq 5\right\}$, where $e_{1}, e_{2}, e_{3} \in E^{1,1}, e_{4} \in E^{2,2}, e_{5} \in E^{2,1}$. The five similitudes are defined by

$$
\begin{array}{lll}
S_{e_{1}}(x)=\rho x, & S_{e_{2}}(x)=r x+\rho(1-r), & S_{e_{3}}(x)=r x+(1-r), \\
S_{e_{4}}(x)=r x+(1-r), & S_{e_{5}}(x)=\rho x, &
\end{array}
$$

where $\rho+2 r-\rho r \leq 1$, i.e., $S_{e_{2}}(1) \leq S_{e_{3}}(0)$ (see Figure 5). For a probability matrix $\left(p_{e}\right)_{e \in E}$, we define

$$
w(k):=p_{e_{1}} \sum_{j=0}^{k} p_{e_{2}}^{j} p_{e_{3}}^{k-j}, \quad k \geq 0 .
$$

Corollary 1.8. Let $\mu=\mu_{1}+\mu_{2}$ be a graph-directed self-similar measure defined by the $G I F S G=(V, E)$ in Example 1.7 together with a probability matrix $\left(p_{e}\right)_{e \in E}$, and let $w(k)$ be defined as in (1.16). Then there exists a unique set of non-negative real numbers $\alpha_{1}, \alpha_{2}$ such that both factors in the following equation are zero:

$$
\left[1-\left(p_{e_{4}} r\right)^{\alpha_{2}}\right]\left[1-\sum_{i=2}^{3}\left(p_{e_{i}} r\right)^{\alpha_{1}}-\left(\prod_{i=2}^{3}\left(1-\left(p_{e_{i}} r\right)^{\alpha_{1}}\right)\right) \cdot \sum_{k=0}^{\infty}\left(w(k) \rho r^{k}\right)^{\alpha_{1}}\right]=0 .
$$

Moreover, $d_{s}=2 \alpha$, where $\alpha:=\max \left\{\alpha_{1}, \alpha_{2}\right\}$.

If $\ell=1,2$,

$$
\lim _{t \rightarrow \infty}\left(e^{-\alpha t} N\left(e^{t},-\Delta_{\left.\mu_{1}\right|_{B_{1, \ell}}}\right)-q_{\ell}(t)\right)=0,
$$

where $q_{\ell}$ is either periodic or non-negative constant depending on whether $\nu_{\ell}$ is lattice or not.

If $\ell=3$ or 4 , then

$$
\lim _{t \rightarrow \infty} e^{-\alpha t} N\left(e^{t},-\Delta_{\left.\mu_{2}\right|_{B_{1, \ell}}}\right)=0 .
$$

Remark 1.9. Let $G=(V, E)$ be defined as in Example 1.7. Let $p_{e_{i}}=\rho=1 / 3$ for $i=1,2,3,4, p_{e_{5}}=2 / 3$, and $r=2 / 7$. The following hold.

(a) Numerical approximations by taking $k$ up to 1000 yield $\alpha_{1}=0.439314 \ldots$, $\alpha_{2}=0$, and hence $d_{s}=0.878628 \ldots$ 
(b) The Hausdorff dimension of the associated graph self-similar set is $d_{f}=$ $0.797012 \ldots$, and moreover, $d_{s}<2 d_{f} /\left(1+d_{f}\right)$.

(c) If $\ell=1$, then there exists a constant $q_{1} \geq 0$ such that

$$
\lim _{t \rightarrow \infty} e^{-\alpha t} N\left(e^{t},-\Delta_{\left.\mu_{1}\right|_{B_{1,1}}}\right)=q_{1}
$$

if $\ell=2$, then there exists a periodic function $q_{2}$ such that

$$
\lim _{t \rightarrow \infty}\left(e^{-\alpha t} N\left(e^{t},-\Delta_{\left.\mu_{1}\right|_{B_{1,2}}}\right)-q_{2}(t)\right)=0 .
$$

In Subsection 6.3, we illustrate Theorem 1.6 by the following example.

Example 1.10. Let $G=(V, E)$ be a GIFS that is not strongly connected with $V=\{1, \ldots, 6\}$ and $E=\left\{e_{i}: 1 \leq i \leq 17\right\}$, where $e_{1}, e_{2}, e_{3} \in E^{1,1}, e_{5}, e_{6} \in E^{2,2}, e_{4} \in E^{2,1}$, $e_{7}, e_{8}, e_{9} \in E^{3,3}, e_{10} \in E^{4,3}, e_{11}, e_{12} \in E^{4,4}, e_{13} \in E^{5,3}, e_{14}, e_{15} \in E^{5,5}, e_{16} \in E^{6,1}$, and $e_{17} \in E^{6,6}$. The 17 similitudes are defined by

$$
\begin{aligned}
& S_{e_{i}}(x)=\rho x, \quad \text { for } i=1,4,7,10,13,16, \\
& S_{e_{j}}(x)=r x+\rho(1-r), \quad \text { for } j=2,5,8,11,14, \\
& S_{e_{k}}(x)=r x+(1-r), \quad \text { for } k=3,6,9,12,15,17,
\end{aligned}
$$

where $\rho+2 r-\rho r \leq 1$, i.e., for $j=2,5,8,11,14, S_{e_{j}}(1) \leq S_{e_{j+1}}(0)$ (see Figure 9 ). For a probability matrix $\left(p_{e}\right)_{e \in E}$ and $k \geq 0$, we define

$$
\begin{array}{ll}
w_{1}(k):=p_{e_{1}} \sum_{j=0}^{k} p_{e_{2}}^{j} p_{e_{3}}^{k-j}, & w_{2}(k):=p_{e_{4}} \sum_{j=0}^{k} p_{e_{5}}^{j} p_{e_{3}}^{k-j}, \\
w_{3}(k):=p_{e_{7}} \sum_{j=0}^{k} p_{e_{8}}^{j} p_{e_{9}}^{k-j}, \quad w_{4}(k):=p_{e_{10}} \sum_{j=0}^{k} p_{e_{11}}^{j} p_{e_{9}}^{k-j}, \\
w_{5}(k):=p_{e_{13}} \sum_{j=0}^{k} p_{e_{14}}^{j} p_{e_{9}}^{k-j} .
\end{array}
$$

Corollary 1.11. Let $\mu=\sum_{i=1}^{6} \mu_{i}$ be the graph-directed self-similar measure defined by the GIFS $G=(V, E)$ in Example 1.10 together with a probability matrix $\left(p_{e}\right)_{e \in E}$. Then there exists a unique set of non-negative real numbers $\alpha_{1}, \ldots, \alpha_{6}$ such that each factor in the following equation equals 0.

$$
\begin{array}{r}
{\left[1-\sum_{i=2}^{3}\left(p_{e_{i}} r\right)^{\alpha_{1}}-\left(\prod_{i=2}^{3}\left(1-\left(p_{e_{i}} r\right)^{\alpha_{1}}\right)\right) \cdot \sum_{k=0}^{\infty}\left(w_{1}(k) \rho r^{k}\right)^{\alpha_{1}}\right] \cdot\left[1-\sum_{i=5}^{6}\left(p_{e_{i}} r\right)^{\alpha_{2}}\right]} \\
(1.20) \cdot\left[1-\sum_{i=8}^{9}\left(p_{e_{i}} r\right)^{\alpha_{3}}-\left(\prod_{i=8}^{9}\left(1-\left(p_{e_{i}} r\right)^{\alpha_{3}}\right)\right) \cdot \sum_{k=0}^{\infty}\left(w_{3}(k) \rho r^{k}\right)^{\alpha_{3}}\right] \cdot\left[1-\sum_{i=11}^{12}\left(p_{e_{i}} r\right)^{\alpha_{4}}\right]
\end{array}
$$




$$
\cdot\left[1-\sum_{i=14}^{15}\left(p_{e_{i}} r\right)^{\alpha_{5}}\right] \cdot\left[1-\left(p_{e_{17}} r\right)^{\alpha_{6}}\right]=0 .
$$

Moreover, $d_{s}=2 \alpha$, where $\alpha:=\max \left\{\alpha_{i}: i=1, \ldots, 6\right\}$. If $\ell=1,2$, there are constants $c_{\ell}>0$ such that

$$
\lim _{t \rightarrow \infty} t^{-1} e^{-\alpha t} N\left(e^{t},-\Delta_{\left.\mu_{1}\right|_{B_{1, \ell}}}\right)=c_{\ell} .
$$

If $\ell=5,6$, there are constants $c_{\ell}>0$ such that

$$
\lim _{t \rightarrow \infty} t^{-2} e^{-\alpha t} N\left(e^{t},-\Delta_{\left.\mu_{3}\right|_{B_{1, \ell}}}\right)=c_{\ell} .
$$

If $\ell=3,4,7,8,9,10,11,12$, then

$$
\lim _{t \rightarrow \infty} e^{-\alpha t} N\left(e^{t},-\Delta_{\left.\mu_{i}\right|_{B_{1, \ell}}}\right)=0 \quad \text { for } \ell \in \Gamma_{i} .
$$

Remark 1.12. Let $G=(V, E)$ be defined as in Example 1.10. Let $r=2 / 7$ and

$$
p_{e_{i}}=1 / 4, \quad p_{e_{j}}=1 / 2, \quad p_{e_{m}}=\rho=1 / 3, \quad p_{e_{n}}=1 / 6,
$$

for $i=1,2,7,8, j=3,9,10,14,16,17, m=4,5,6,11,15$, and $n=12,13$. The following hold.

(a) Numerical approximations by taking $k$ up to 1000 yield $\alpha_{1}=0.435715 \ldots$, $\alpha_{2}=0.294784 \ldots, \alpha_{3}=0.435715 \ldots, \alpha_{4}=0.258401 \ldots, \alpha_{5}=0.323599 \ldots, \alpha_{6}=0$. Hence $\alpha=$ $0.435715 \ldots$, and $d_{s}=0.871430 \ldots$

(b) The Hausdorff dimension of the associated graph self-similar set is $d_{f}=$ $0.797012 \ldots$, and moreover, $d_{s}<2 d_{f} /\left(1+d_{f}\right)$.

The rest of this paper is organized as follows. In Section 2, we give a modified version of the definition of (EFT). In Section 3, we introduce some properties of the eigenvalue counting function. In Section 4, we derive renewal equations and prove Theorems 1.1 and 1.6. Section 5 illustrates Theorem 1.1 by the strongly connected GIFS defined in Example 1.2; we also prove Corollary 1.3. In Section 6, we study GIFSs in Examples 1.7 and 1.10, which are not strongly connected. We also prove Corollaries 1.8 and 1.11 .

\section{Graph-directed iterated function systems and measures essentially of finite type}

\subsection{Graph-directed iterated function systems}

A graph-directed iterated function system (GIFS) of contractive similitudes is an ordered pair $G=(V, E)$ described as follows (see [13]). $V$ is a set of vertices 
labeled by $\{1, \ldots, q\} . E$ is a set of directed edges, each beginning and ending at a vertex. It is possible for an edge to begin and end at the same vertex and we allow more than one edge between two vertices. To each edge $e \in E$, there corresponds a contractive similitude $S_{e}(x): \mathbf{R}^{d} \rightarrow \mathbf{R}^{d}$ defined as

$$
S_{e}(x)=\rho_{e} R_{e} x+b_{e},
$$

where $\rho_{e} \in(0,1)$ is the contraction ratio, $R_{e}$ is an orthogonal transformation, and $b_{e} \in \mathbf{R}^{d}$. Let $E^{i, j}$ denote the set of all edges that begin at vertex $i$ and end at vertex $j$. We call $\boldsymbol{e}=e_{1} \ldots e_{k}$ a path (or an $\boldsymbol{e}$-path) with length $k$, if the terminal vertex of each edge $e_{i}(1 \leq i \leq k-1)$ equals the initial vertex of the edge $e_{i+1}$. It is well known that there exists a unique family of nonempty compact sets $K_{1}, \ldots, K_{q}$ satisfying

$$
K_{i}=\bigcup_{j=1}^{q} \bigcup_{e \in E^{i, j}} S_{e}\left(K_{j}\right), \quad i=1, \ldots, q
$$

Define

$$
K:=\bigcup_{i=1}^{q} K_{i}
$$

We call $K$ the graph self-similar set associated with $G=(V, E)$. Assume that for each edge $e \in E$, there corresponds a transition probability $p_{e}>0$, and the weights of all edges leaving a given vertex $i$ sum to 1 , namely,

$$
\sum_{j \in V} \sum_{e \in E^{i, j}} p_{e}=1
$$

Then for each $i \in V$, there exists a unique Borel probability measures $\mu_{i}$ such that

$$
\mu_{i}=\sum_{j=1}^{q} \sum_{e \in E^{i, j}} p_{e} \mu_{j} \circ S_{e}^{-1}
$$

We note that $\operatorname{supp}\left(\mu_{i}\right)=K_{i}$ for all $i \in V$. Finally, let $\mu:=\sum_{i=1}^{q} \mu_{i}$ and call it a graph-directed self-similar measure. We say that $G=(V, E)$ satisfies the graph open set condition (GOSC) (see [23]) if there exists a family $\left\{O_{i}\right\}_{i=1}^{q} \subseteq \mathbf{R}^{d}$ of nonempty bounded open sets such that for all $i, j, j^{\prime} \in V$,

$$
\bigcup_{e \in E^{i, j}} S_{e}\left(O_{j}\right) \subseteq O_{i} \text { and } S_{e}\left(O_{j}\right) \cap S_{e^{\prime}}\left(O_{j^{\prime}}\right)=\varnothing \text { for all distinct } e \in E^{i, j} \text { and } e^{\prime} \in E^{i, j^{\prime}} \text {. }
$$

It is obvious that $K_{i} \subseteq \bar{O}_{i}$, i.e., $\operatorname{supp}\left(\mu_{i}\right) \subseteq \bar{O}_{i}$. A GIFS, as well as any associated graph-directed self-similar measure, are said to have overlaps if (GOSC) fails. Let 
$\left\{\Omega_{i}\right\}_{i=1}^{q}$ be a family of nonempty bounded open subsets of $\mathbf{R}^{d}$. We say that $\left\{\Omega_{i}\right\}_{i=1}^{q}$ is invariant under the GIFS $G=(V, E)$ if $\bigcup_{e \in E^{i, j}} S_{e}\left(\Omega_{j}\right) \subseteq \Omega_{i}$ for $i=1, \ldots, q$. We say $G$ is connected if for each pair of vertices $i, j \in V$, there is a (non-directed) path between them. $G$ is said to be strongly connected if for each pair of vertices $i, j \in V$, there is a directed path from $i$ to $j$. A strongly connected component of $G$ is a maximal subgraph $H$ of $G$ such that $H$ is strongly connected. Strongly connected components are pairwise disjoint and do not necessarily cover $G$. A single vertex may be a strongly connected component if it loops to itself. In this paper, we assume that each graph has at least one strongly connected component.

\subsection{The essentially finite type condition for graph-directed self-similar measures}

Let $\Omega \subseteq \mathbf{R}^{d}$ be a bounded open subset and $\mu$ be a positive finite Borel measure with $\operatorname{supp}(\mu) \subseteq \bar{\Omega}$ and $\mu(\Omega)>0$. We call a $\mu$-measurable subset $U$ of $\Omega$ is a cell (in $\Omega)$ if $\mu(U)>0$. Clearly, $\Omega$ itself is a cell.

We say that two cells $U$ and $U^{\prime}$ are $\mu$-equivalent, denoted by $U \simeq_{\mu, \tau, w} U^{\prime}$ (or simply $\left.U \simeq{ }_{\mu} U^{\prime}\right)$, if there exist some similitude $\tau: U \rightarrow U^{\prime}$ and some constant $w>0$ such that $\tau(U)=U^{\prime}$ and

$$
\left.\mu\right|_{U^{\prime}}=\left.w \mu\right|_{U} \circ \tau^{-1}
$$

It is easy to check that $\simeq_{\mu}$ is an equivalence relation.

Two cells $U, U^{\prime}$ in $\Omega$ are measure disjoint with respect to $\mu$ if $\mu\left(U \cap U^{\prime}\right)=0$. We call a finite family $\mathbf{P}$ of measure disjoint cells a $\mu$-partition of $\Omega$ if $U \subseteq \Omega$ for all $U \in \mathbf{P}$, and $\mu(\Omega)=\sum_{U \in \mathbf{P}} \mu(U)$. A sequence of $\mu$-partitions $\left\{\mathbf{P}_{k}\right\}_{k \geq 1}$ is refining if for any $U^{\prime} \in \mathbf{P}_{k}$ and any $U \in \mathbf{P}_{k+1}$, either $U \subseteq U^{\prime}$ or they are measure disjoint, i.e., each member of $\mathbf{P}_{k+1}$ is a subset of some member of $\mathbf{P}_{k}$.

Let $\mathbf{B}:=\left\{B_{1, \ell}\right\}_{\ell \in \Gamma}$ be a finite family of measure disjoint cells in $\Omega$, and for each $\ell \in \Gamma$, let $\left\{\mathbf{P}_{k, \ell}\right\}_{k \geq 1}$ be a family of refining $\mu$-partitions of $B_{1, \ell}$ with $\mathbf{P}_{1, \ell}:=\left\{B_{1, \ell}\right\}$, where $\Gamma$ is a finite index set. We divide each $\mathbf{P}_{k, \ell}, k \geq 2$, into two (possibly empty) subcollections, $\mathbf{P}_{k, \ell}^{1}$ and $\mathbf{P}_{k, \ell}^{2}$, with respect to $\mathbf{B}$, defined as follows:

$$
\begin{aligned}
& \mathbf{P}_{k, \ell}^{1}:=\left\{B \in \mathbf{P}_{k, \ell}: B \simeq{ }_{\mu} B_{1, i} \text { for some } i \in \Gamma\right\}, \\
& \mathbf{P}_{k, \ell}^{2}:=\mathbf{P}_{k, \ell} \backslash \mathbf{P}_{k, \ell}^{1}=\left\{B \in \mathbf{P}_{k, \ell}: B \notin \mathbf{P}_{k, \ell}^{1}\right\} .
\end{aligned}
$$

Definition 2.1. We say that a graph-directed self-similar measure $\mu=\sum_{i=1}^{q} \mu_{i}$ on $\mathbf{R}^{d}$ is essentially of finite type (EFT) if there exist a family of bounded open subsets $\left\{\Omega_{i}\right\}_{i=1}^{q}$ with $\Omega_{i} \subseteq \mathbf{R}^{d}, \operatorname{supp}\left(\mu_{i}\right) \subseteq \bar{\Omega}_{i}$ and $\mu\left(\Omega_{i}\right)>0$, and a finite family $\mathbf{B}:=$ $\left\{B_{1, \ell}\right\}_{\ell \in \Gamma}$ of measure disjoint cells, $B_{1, \ell} \subseteq \Omega_{i_{\ell}}$ for some $i_{\ell}=1, \ldots, q$, such that for any 
$\ell \in \Gamma$, there is a family of $\mu$-partitions $\left\{\mathbf{P}_{k, \ell}\right\}_{k \geq 1}$ of $B_{1, \ell}$ satisfying the following conditions:

(1) $\mathbf{P}_{1, \ell}=\left\{B_{1, \ell}\right\}$, and there exists some $B \in \mathbf{P}_{2, \ell}^{1}$ such that $B \neq B_{1, \ell}$;

(2) if for some $k \geq 2$, there exists some $B \in \mathbf{P}_{k, \ell}^{1}$, then $B \in \mathbf{P}_{k+1, \ell}^{1}$ and hence $B \in \mathbf{P}_{m, \ell}^{1}$ for all $m \geq k$;

(3) $\lim _{k \rightarrow \infty} \sum_{B \in \mathbf{P}_{k, \ell}^{2}} \mu(B)=0$.

Here $\mathbf{P}_{k, \ell}^{1}$ and $\mathbf{P}_{k, \ell}^{2}(k \geq 2)$ are defined as in (2.6). In this case, we call $\left\{\Omega_{i}\right\}_{i=1}^{q}$ an EFT-family, B a basic family of cells, and $(\mathbf{B}, \mathbf{P}):=\left(\left\{B_{1, \ell}\right\},\left\{\mathbf{P}_{k, \ell}\right\}_{k \geq 1}\right)_{\ell \in \Gamma}$ a basic pair.

For $k \geq 2$ and $\ell \in \Gamma$, let $\mathbf{P}_{k, \ell}=\left\{B_{k, \ell, i}, i=1,2, \ldots\right\}$. For $B_{k, \ell, i}$, the subscript $i$ denotes the $i$-th measure disjoint cell of the $\mu$-partition $\mathbf{P}_{k . \ell}$.

Definition 2.2. Assume that a graph-directed self-similar measure $\mu=$ $\sum_{i=1}^{q} \mu_{i}$ satisfies (EFT) with $\left\{\Omega_{i}\right\}_{i=1}^{q}$ being an EFT-family and $(\mathbf{B}, \mathbf{P}):=$ $\left(\left\{B_{1, \ell}\right\},\left\{\mathbf{P}_{k, \ell}\right\}_{k \geq 1}\right)_{\ell \in \Gamma}$ being a basic pair. We say that $(\mathbf{B}, \mathbf{P})$ is regular if each cell $B \in \bigcup_{k>1, \ell \in \Gamma} \mathbf{P}_{k, \ell}$ is connected, and for any $\ell \in \Gamma$, there exist some similitude $\tau_{\ell}$, some $\Omega_{j_{\ell}}$ and some constant $w(\ell)>0$ such that $\tau_{\ell}\left(\Omega_{j_{\ell}}\right) \subseteq B_{1, \ell}$ and $\mu \geq w(\ell) \mu \circ \tau_{\ell}^{-1}$ on $\tau_{\ell}\left(\Omega_{j_{\ell}}\right)$. In this case, we call $\mathbf{B}$ a regular basic family of cells.

\section{Eigenvalue counting function}

\subsection{Eigenvalue counting function on $\mathbf{R}$ ([19, Section 4.1])}

In this subsection, we only consider one-dimensional Laplacians. Let $(\mathcal{E}, \operatorname{dom} \mathcal{E})$ be defined as in (1.4) with $\Omega=(a, b)$ and let $-\Delta_{\mu}$ be the associated Dirichlet Laplacian on $L^{2}((a, b), \mu)$. Let $\mathcal{P}=\left\{a_{i}\right\}_{i=0}^{n+1}$ be a partition of $[a, b]$ satisfying

$$
a_{0}:=a<a_{1}<\ldots<a_{n+1}=: b .
$$

Define $\mathcal{F}:=\mathcal{F}(\mathcal{P})=\left\{u \in \operatorname{dom} \mathcal{E}: u\left(a_{i}\right)=0\right.$ for all $\left.i=0, \ldots, n+1\right\}$. Then $\mathcal{F}$ is a closed subspace of $\operatorname{dom} \mathcal{E}$. Define a relation $\sim_{\mathcal{E}}$ on $\operatorname{dom} \mathcal{E}$, induced by $\mathcal{F}$, by $u \sim_{\mathcal{E}} v$ if and only if $u-v \in \mathcal{F}$. Then $\sim_{\mathcal{E}}$ is an equivalence relation on $\operatorname{dom} \mathcal{E}$. Define the quotient space

$$
\operatorname{dom} \mathcal{E} / \mathcal{F}:=\left\{[u]_{\mathcal{E}}: u \in \operatorname{dom} \mathcal{E}\right\}
$$

where $[u]_{\mathcal{E}}$ is the equivalence class of $u$. Define addition and scalar multiplication on $\operatorname{dom} \mathcal{E} / \mathcal{F}$ as usual. For each $i=1, \ldots, n$, let $f_{i}$ be a function in $\operatorname{dom} \mathcal{E}$ that satisfies

$$
f_{i}\left(a_{j}\right)=\delta_{i j}, \quad i, j=1, \ldots, n,
$$


where $\delta_{i j}$ is the Kronecker delta. Such an $f_{i}$ clearly exists. It is easy to prove that

$$
\operatorname{dom} \mathcal{E} / \mathcal{F}=\operatorname{span}\left\{\left[f_{i}\right]_{\mathcal{E}}: i=1, \ldots, n\right\} \quad \text { and } \quad \operatorname{dim}(\operatorname{dom} \mathcal{E} / \mathcal{F})=n .
$$

Let $-\Delta_{\left.\mu\right|_{(a, b)}}^{\mathcal{F}}$ be the Laplacian defined by the Dirichlet form (1.4) with $\operatorname{dom} \mathcal{E}=\mathcal{F}$, and let $N\left(\lambda,-\Delta_{\left.\mu\right|_{(a, b)}}^{\mathcal{F}}\right):=\#\left\{n: \lambda_{n}\left(-\Delta_{\left.\mu\right|_{(a, b)}}^{\mathcal{F}}\right) \leq \lambda\right\}$ be the associated eigenvalue counting function. If $\mathcal{F}=\mathcal{N}^{\perp}$, where $\mathcal{N}$ is defined as in Section 1 , then $N\left(\lambda,-\Delta_{\left.\mu\right|_{(a, b)}}^{\mathcal{F}}\right)$ reduces to $N\left(\lambda,-\Delta_{\left.\mu\right|_{(a, b)}}\right)$. It follows from the variational formula that

$$
N\left(\lambda,-\Delta_{\left.\mu\right|_{(a, b)}}^{\mathcal{F}}\right) \leq N\left(\lambda,-\Delta_{\left.\mu\right|_{(a, b)}}\right) \leq N\left(\lambda,-\Delta_{\left.\mu\right|_{(a, b)}}^{\mathcal{F}}\right)+\# \mathcal{P}-2
$$

If $\operatorname{supp}(\mu)=[a, b]$, then $N\left(\lambda,-\Delta_{\left.\mu\right|_{(a, b)}}^{\mathcal{F}}\right)=\sum_{i=0}^{n} N\left(\lambda,-\Delta_{\left.\mu\right|_{\left(a_{i}, a_{i+1}\right)}}\right)$. Next, we state a similar formula. A proof can be found in [19, Proposition 4.1].

Proposition 3.1. Let $\mu$ be a continuous positive finite Borel measure on $[a, b]$ with $\operatorname{supp}(\mu) \subseteq[a, b]$. Suppose there exists a nonempty subset $\Lambda \subseteq\{0,1, \ldots, n\}$ such that $\mu\left(a_{i}, a_{i+1}\right)>0$ for any $i \in \Lambda$ and $\mu\left(a_{j}, a_{j+1}\right)=0$ for any $j \notin \Lambda$. Then

$$
N\left(\lambda,-\Delta_{\left.\mu\right|_{(a, b)}}^{\mathcal{F}}\right)=\sum_{i \in \Lambda} N\left(\lambda,-\Delta_{\left.\mu\right|_{\left(a_{i}, a_{i+1}\right)}}\right) .
$$

\subsection{Unitarily equivalent operators}

In this subsection, we state a slightly modified version of $[17$, Propositions 2.2 and 2.3] below.

Proposition 3.2. ([17, Proposition 2.2]) Let $S: \mathbf{R} \rightarrow \mathbf{R}$ be a similitude, with Lipschitz constant $r$, such that $S(a, b)=(c, d)$. Let $\mu$ be a continuous positive finite Borel measure on $[a, b]$ with $\operatorname{supp}(\mu) \subseteq[a, b]$. Then

(a) $-\Delta_{\left.\mu \circ S^{-1}\right|_{(c, d)}}$ and $r^{-1} \cdot\left(-\Delta_{\left.\mu\right|_{(a, b)}}\right)$ are unitarily equivalent.

(b) If, in addition, $\left.\mu\right|_{(c, d)}=w \mu \circ S^{-1}$ on $(c, d)$ for some constant $w>0$, then $-\Delta_{\left.\nu\right|_{(c, d)}}$ and $(r w)^{-1} \cdot\left(-\Delta_{\left.\mu\right|_{(a, b)}}\right)$ are unitarily equivalent.

Note that unitarily equivalent operators have the same set of eigenvalues.

Proposition 3.3. ([17, Proposition 2.3]) Let $\mu, \nu$ be continuous positive finite Borel measures on $[a, b]$ and assume that there exists some constant $w>0$ such that $\mu \leq w \nu$ on $[a, b]$. Then for any $n \geq 1, \lambda_{n}\left(-\Delta_{\mu}\right) \geq w^{-1} \cdot \lambda_{n}\left(-\Delta_{\nu}\right)$.

The following result follows by combining Propositions 3.2 and 3.3. The proof can be found in [19]. 
Proposition 3.4. Let $\mu$ be a continuous positive finite Borel measure on $\mathbf{R}$ and assume that there exist a similitude $S$ with Lipschitz constant $r$, and a constant $w>0$ such that $S([a, b])=[c, d]$ and $\mu \geq w \mu \circ S^{-1}$ on $[c, d]$. Then $N\left(w r \lambda,-\Delta_{\left.\mu\right|_{(a, b)}}\right) \leq$ $N\left(\lambda,-\Delta_{\left.\mu\right|_{(c, d)}}\right)$.

\section{Renewal equation and proofs of Theorems 1.1 and 1.6}

\subsection{Renewal equation}

Let $\mu=\sum_{i=1}^{q} \mu_{i}$ be a graph-directed self-similar measure defined by $G=(V, E)$ on $\mathbf{R}$. In the rest of this section, we assume that $\mu$ satisfies (EFT) with $\left\{\Omega_{i}\right\}_{i=1}^{q}$ being an EFT-family, with $(\mathbf{B}, \mathbf{P}):=\left(\left\{B_{1, \ell}\right\},\left\{\mathbf{P}_{k, \ell}\right\}_{k \geq 1}\right)_{\ell \in \Gamma}$ being a regular basic pair. The regularity of $(\mathbf{B}, \mathbf{P})$ implies that each cell $B \in \bigcup_{k \geq 1, \ell \in \Gamma} \mathbf{P}_{k, \ell}$ is an interval. This allows us to apply Propositions 3.1-3.4. For $i \in V$, let $\Gamma_{i}$ be defined as in (1.7) and

$$
\mathbf{B}_{i}:=\left\{B_{1, \ell}: \ell \in \Gamma_{i}\right\}
$$

Then $\Gamma=\bigcup_{i=1}^{q} \Gamma_{i}$ and $\mathbf{B}=\bigcup_{i=1}^{q} \mathbf{B}_{i}$. Note that $\Gamma_{i}$ and $\mathbf{B}_{i}$ maybe empty. The following Proposition has been modified from [19, Proposition 4.5] to suit our purpose. The proof is similar.

Proposition 4.1. Let $\mu=\sum_{i=1}^{q} \mu_{i}$ be a graph-directed self-similar measure defined by a $G I F S G=(V, E)$ on $\mathbf{R}$. Assume that $\mu$ satisfies $(E F T)$ with $\left\{\Omega_{i}\right\}_{i=1}^{q}$ being an EFT-family and with $\mathbf{B}:=\left\{B_{1, \ell}: \ell \in \Gamma\right\}$ being a regular basic family of cells. Let $\Omega=\bigcup_{i=1}^{q} \Omega_{i}$, and $\Gamma_{i}, \mathbf{B}_{i}$ defined as in (4.1). Then for $i \in V$ and any $\ell \in \Gamma_{i}$, there exists some constant $\bar{c}_{\ell}>0$ such that

$$
N\left(\lambda,-\Delta_{\left.\mu_{i}\right|_{B_{1, \ell}}}\right) \leq N\left(\lambda,-\Delta_{\left.\mu\right|_{\Omega}}\right) \leq N\left(\bar{c}_{\ell} \lambda,-\Delta_{\left.\mu_{i}\right|_{B_{1, \ell}}}\right) .
$$

Proposition 4.1 implies that the asymptotic behavior of $N\left(\lambda,-\Delta_{\mu}\right)$ is controlled by that of $N\left(\lambda,-\Delta_{\left.\mu_{i}\right|_{B_{1, \ell}}}\right)$ for $i \in V$ and $\ell \in \Gamma_{i}$.

Step 1. Derivation of functional equations. For $\ell \in \Gamma_{i}$ and $k \geq 2$, let $\mathbf{P}_{k, \ell}^{1}$ and $\mathbf{P}_{k, \ell}^{2}$ be defined as in (2.6) with respect to $\mathbf{B}$, where $i \in V$. Without loss of generality, we may assume that $\Gamma_{i}$ can be partitioned into two (possibly empty) sub-collections, $\Gamma_{i}^{\prime}$ and $\Gamma_{i}^{*}$, defined as follows. An index $\ell \in \Gamma_{i}$ belongs to $\Gamma_{i}^{\prime}$ if there exists some integer $k$ satisfying $\mathbf{P}_{k, \ell}^{2}=\varnothing$. Let $\varkappa_{\ell} \geq 2$ (depending on $\ell$ ) denote the smallest of such $k$. Define $\Gamma_{i}^{*}:=\Gamma_{i} \backslash \Gamma_{i}^{\prime}$ and let $\varkappa_{\ell}:=\infty$ for $\ell \in \Gamma_{i}^{*}$. Let $\Gamma^{\prime}=\bigcup_{i=1}^{q} \Gamma_{i}^{\prime}$ and $\Gamma^{*}=\bigcup_{i=1}^{q} \Gamma_{i}^{*}$. Then $\Gamma=\Gamma^{\prime} \cup \Gamma^{*}$. 
For $i \in V$, fix any $\ell \in \Gamma_{i}$. The definition of (EFT) implies that for any $2 \leq k \leq \varkappa_{\ell}$, there exist two finite disjoint $G_{k, \ell}^{1}, G_{k, \ell}^{2} \subseteq \mathbf{N}$ such that

$$
\mathbf{P}_{k, \ell}^{1}=\bigcup_{m=2}^{k}\left\{B_{m, \ell, p}: p \in G_{m, \ell}^{1}\right\} \quad \text { and } \quad \mathbf{P}_{k, \ell}^{2}=\left\{B_{k, \ell, p}: p \in G_{k, \ell}^{2}\right\}
$$

Condition (1) of (EFT) implies that $G_{2, \ell}^{1} \neq \varnothing$. If $\ell \in \Gamma^{*}$, condition (3) of (EFT) implies that $\lim _{k \rightarrow \infty} \sum_{p \in G_{k, \ell}^{2}} \mu\left(B_{k, \ell, p}\right)=0$.

Proposition 4.2. Assume that $\mu$ satisfies $(E F T)$. Let $\ell \in \Gamma_{i}$, and

$$
J_{\ell}:=\left\{j \in V: S_{e}\left(\Omega_{j}\right) \subseteq B_{1, \ell} \text { for } e \in E^{i, j}\right\},
$$

where $i \in V$. Let $2 \leq k \leq \varkappa_{\ell}$. If $G_{k, \ell}^{1} \neq \varnothing$, then for each $p \in G_{k, \ell}^{1}$, there exist some $\xi(k, \ell, p)>0$ and $c(k, \ell, p) \in \Gamma_{j}, j \in J_{\ell}$, such that

$$
N\left(\lambda,-\Delta_{\left.\mu_{i}\right|_{B_{k, \ell, p}}}\right)=N\left(\xi(k, \ell, p) \lambda,-\Delta_{\left.\mu_{j}\right|_{B_{1, c(k, \ell, p)}}}\right) .
$$

Proof. For any $p \in G_{k, \ell}^{1}$, by the definition of $\mathbf{P}_{k, \ell}^{1}$, there exist some similitude $S_{e(k, \ell, p)}$ with Lipschitz constant $r_{e(k, \ell, p)}$, as well as constants $w(k, \ell, p)>0$ and $c(k, \ell, p) \in \Gamma_{j}$ such that $\left.\mu_{i}\right|_{B_{k, \ell, p}}=\left.w(k, \ell, p) \mu_{j}\right|_{B_{1, c(k, \ell, p)}} \circ S_{e(k, \ell, p)}^{-1}$, where $j \in J_{\ell}$. Combining this with Proposition 3.2(b), we get (4.4) with $\xi(k, \ell, p):=w(k, \ell, p) r_{e(k, \ell, p)}$. This completes the proof.

For all $i \in V$, each $\ell \in \Gamma_{i}$, and $1 \leq n \leq \varkappa_{\ell}$, we define a partition $\mathcal{P}_{n, \ell}$ of $B_{1, \ell}$ as follows:

$$
\mathcal{P}_{n, \ell}:=\left\{x: x \text { is an end-point of some interval in } \mathbf{P}_{n, \ell}\right\},
$$

and let $\mathcal{F}_{n, \ell}:=\mathcal{F}\left(\mathcal{P}_{n, \ell}\right)$. Note that for any $i \in V$, any $\ell \in \Gamma_{i}$, and $2 \leq n \leq \varkappa_{\ell}$, we have $\# \mathcal{P}_{n, \ell} \leq 2 \# \mathbf{P}_{n, \ell}$. It follows from Proposition 3.1 that for $i \in V, \ell \in \Gamma_{i}$, and $2 \leq n \leq \varkappa_{\ell}$,

$$
N\left(\lambda,-\Delta_{\left.\mu_{i}\right|_{B_{1, \ell}}}^{\mathcal{F}_{n, \ell}}\right)=\sum_{k=2}^{n} \sum_{p \in G_{k, \ell}^{1}} N\left(\lambda,-\Delta_{\left.\mu_{i}\right|_{B_{k, \ell, p}}}\right)+\sum_{p \in G_{n, \ell}^{2}} N\left(\lambda,-\Delta_{\left.\mu_{i}\right|_{B_{n}, \ell, p}}\right) .
$$

Combining this with (3.1) and Proposition 4.2, for any $\ell \in \Gamma_{i}^{\prime}$,

$$
N\left(\lambda,-\Delta_{\left.\mu_{i}\right|_{B_{1, \ell}}}\right)=\sum_{k=2}^{\varkappa_{\ell}} \sum_{p \in G_{k, \ell}^{1}} N\left(\xi(k, \ell, p) \lambda,-\Delta_{\left.\mu_{j}\right|_{B_{1, c(k, \ell, p)}}}\right)+\varepsilon\left(\varkappa_{\ell}, \ell\right),
$$


where $0 \leq \varepsilon\left(\varkappa_{\ell}, \ell\right) \leq 2 \# \mathbf{P}_{\varkappa_{\ell}, \ell}-2$. Similarly, for $\ell \in \Gamma_{i}^{*}$ and $n \geq 2$, we have

$$
\begin{aligned}
N\left(\lambda,-\Delta_{\left.\mu_{i}\right|_{B_{1}, \ell}}\right)= & \sum_{k=2}^{n} \sum_{p \in G_{k, \ell}^{1}} N\left(\xi(k, \ell, p) \lambda,-\Delta_{\left.\mu_{j}\right|_{B_{1, c(k, \ell, p)}}}\right) \\
& +\sum_{p \in G_{n, \ell}^{2}} N\left(\lambda,-\Delta_{\left.\mu_{i}\right|_{B_{n, \ell, p}}}\right)+\varepsilon(n, \ell),
\end{aligned}
$$

where $0 \leq \varepsilon(n, \ell) \leq 2 \# \mathbf{P}_{n, \ell}-2$.

Step 2. Derivation of the vector-valued equation.

Case 1. ( $G$ is strongly connected) In this case, $\eta=1$. For each $i \in V$, each $\ell \in \Gamma_{i}$, and $\alpha>0$, define

$$
f_{\ell}(t)=f_{\ell}^{(\alpha)}(t):=e^{-\alpha t} N\left(e^{t},-\Delta_{\left.\mu_{i}\right|_{B_{1, \ell}}}\right), \quad t \in \mathbf{R} .
$$

Let $\lambda=e^{t}$. Then $e^{-\alpha t} N\left(\beta \lambda,-\Delta_{\left.\mu_{i}\right|_{B_{1, \ell}}}\right)=\beta^{\alpha} f_{\ell}(t+\ln \beta)$ for any $\beta>0$. Now, multiply both sides of (4.5) and (4.6) by $e^{-\alpha t}$. Then for $\ell \in \Gamma_{i}^{\prime}$, we have

$$
f_{\ell}(t)=\sum_{k=2}^{\varkappa_{\ell}} \sum_{p \in G_{k, \ell}^{1}} \xi(k, \ell, p)^{\alpha} f_{c(k, \ell, p)}(t+\ln \xi(k, \ell, p))+z_{\ell}^{(\alpha)}(t),
$$

where $z_{\ell}^{(\alpha)}(t):=e^{-\alpha t} \varepsilon\left(\varkappa_{\ell}, \ell\right)$. For $\ell \in \Gamma_{i}^{*}$ and $n \geq 2$, we obtain

$$
\begin{aligned}
f_{\ell}(t)= & \sum_{k=2}^{\infty} \sum_{p \in G_{k, \ell}^{1}} \xi(k, \ell, p)^{\alpha} f_{c(k, \ell, p)}(t+\ln \xi(k, \ell, p))+z_{\ell}^{(\alpha)}(t) \\
& -\sum_{k=n+1}^{\infty} \sum_{p \in G_{k, \ell}^{1}} \xi(k, \ell, p)^{\alpha} f_{c(k, \ell, p)}(t+\ln \xi(k, \ell, p)),
\end{aligned}
$$

where

$$
z_{\ell}^{(\alpha)}(t):=e^{-\alpha t}\left(\sum_{p \in G_{n, \ell}^{2}} N\left(\lambda,-\Delta_{\left.\mu_{i}\right|_{B_{n, \ell, p}}}\right)+\varepsilon(n, \ell)\right) .
$$

Since $\lambda_{1}\left(-\Delta_{\left.\mu_{i}\right|_{B_{1, \ell}}}\right)>0$ for any $i \in V$ and any $\ell \in \Gamma_{i}$, there exists $t_{0} \in \mathbf{R}$ such that $f_{\ell}(t)=0$ for any $t<t_{0}$ and any $\ell \in \Gamma_{i}$. For $t \in \mathbf{R}, i \in V$ and $\ell \in \Gamma_{i}^{*}$, let $n_{t}:=n_{t}(\ell)$ be the positive integer such that

$$
t+\max \left\{\ln \xi(k, \ell, p): p \in G_{k, \ell}^{1}\right\}<t_{0} \quad \text { for all } k>n_{t} .
$$


Let $n=n_{t}$ in (4.9). Then

$$
f_{\ell}(t)=\sum_{k=2}^{\infty} \sum_{p \in G_{k, \ell}^{1}} \xi(k, \ell, p)^{\alpha} f_{c(k, \ell, p)}(t+\ln \xi(k, \ell, p))+z_{\ell}^{(\alpha)}(t) \quad \text { for } \ell \in \Gamma_{i}^{*},
$$

where $z_{\ell}^{(\alpha)}(t)$ is obtained from that in (4.10) by replacing $n$ with $n_{t}$. For $i \in V, \ell \in \Gamma_{i}$, let $\mu_{\ell \ell^{\prime}}^{(\alpha)}$ be the discrete measure such that for $2 \leq k \leq \varkappa_{\ell}, p \in G_{k, \ell}^{1}, \ell^{\prime}=c(k, \ell, p) \in \Gamma_{j}$ and $j \in J_{\ell}$,

$$
\mu_{\ell \ell^{\prime}}^{(\alpha)}(-\ln \xi(k, \ell, p)):=\xi(k, \ell, p)^{\alpha} .
$$

Then (see (1.10))

$$
\mu_{\ell \ell^{\prime}}^{(\alpha)}(\mathbf{R})=\sum_{k=2}^{\varkappa_{\ell}} \sum_{p \in G_{k, \ell}^{1}} \xi(k, \ell, p)^{\alpha} \quad \text { and } \quad F_{\ell}(\alpha)=\sum_{\ell^{\prime} \in \Gamma} \sum_{k=2}^{\varkappa_{\ell}} \sum_{p \in G_{k, \ell}^{1}} \xi(k, \ell, p)^{\alpha} .
$$

Case 2. ( $G$ is not strongly connected) If $G=(V, E)$ is not strongly connected, then there exists some $i, j \in V$ satisfying $E^{i, j}=\varnothing$. That is, $\bigcup_{\ell \in \Gamma_{i}} J_{\ell}=\varnothing$. Assume that $G$ has $\eta$ strongly connected components. For $m=1, \ldots, \eta$, let $\mathcal{S C}_{m}$ be defined as in (1.6).

For $m=1, \ldots, \eta$, each $i \in \mathcal{S C}_{m}$ and each $\ell \in \Gamma_{i}$, define

$$
f_{\ell}(t)=f_{\ell}^{\left(\alpha_{m}\right)}(t):=e^{-\alpha_{m} t} N\left(e^{t},-\Delta_{\left.\mu_{i}\right|_{B_{1, \ell}}}\right), \quad \alpha_{m}>0, t \in \mathbf{R} .
$$

Let $\lambda=e^{t}$. Then $e^{-\alpha_{m} t} N\left(\beta \lambda,-\Delta_{\left.\mu_{i}\right|_{B_{1, \ell}}}\right)=\beta^{\alpha_{m}} f_{\ell}(t+\ln \beta)$ for any $\beta>0$. Now, multiply both sides of (4.5) by $e^{-\alpha_{m} t}$. Then for $\ell \in \Gamma_{i}^{\prime}$, we have

$$
f_{\ell}(t)=\sum_{k=2}^{\varkappa_{\ell}} \sum_{p \in G_{k, \ell}^{1}} \xi(k, \ell, p)^{\alpha_{m}} f_{c(k, \ell, p)}(t+\ln \xi(k, \ell, p))+z_{\ell}^{\left(\alpha_{m}\right)}(t),
$$

where $z_{\ell}^{\left(\alpha_{m}\right)}(t):=e^{-\alpha_{m} t} \varepsilon\left(\varkappa_{\ell}, \ell\right)$. Similarly, for $\ell \in \Gamma_{i}^{*}$ and $n \geq 2$, we obtain

$$
\begin{aligned}
f_{\ell}(t)= & \sum_{k=2}^{\infty} \sum_{p \in G_{k, \ell}^{1}} \xi(k, \ell, p)^{\alpha_{m}} f_{c(k, \ell, p)}(t+\ln \xi(k, \ell, p))+z_{\ell}^{\left(\alpha_{m}\right)}(t) \\
& -\sum_{k=n+1}^{\infty} \sum_{p \in G_{k, \ell}^{1}} \xi(k, \ell, p)^{\alpha_{m}} f_{c(k, \ell, p)}(t+\ln \xi(k, \ell, p))
\end{aligned}
$$

where

$$
z_{\ell}^{\left(\alpha_{m}\right)}(t):=e^{-\alpha_{m} t}\left(\sum_{p \in G_{n, \ell}^{2}} N\left(\lambda,-\Delta_{\left.\mu_{i}\right|_{B_{n, \ell, p}}}\right)+\varepsilon(n, \ell)\right)
$$


Since $\lambda_{1}\left(-\Delta_{\left.\mu_{i}\right|_{B_{1, \ell}}}\right)>0$ for all $i \in V$ and all $\ell \in \Gamma_{i}$, there exists $t_{0} \in \mathbf{R}$ such that $f_{\ell}(t)=$ 0 for any $t<t_{0}$ and any $\ell \in \Gamma_{i}$. For $t \in \mathbf{R}, i \in V$ and $\ell \in \Gamma_{i}^{*}$, let $n_{t}:=n_{t}(\ell)$ be the positive integer such that

$$
t+\max \left\{\ln \xi(k, \ell, p): p \in G_{k, \ell}^{1}\right\}<t_{0} \quad \text { for all } k>n_{t} .
$$

Let $n=n_{t}$ in (4.17). Then for $m=1, \ldots, \eta, i \in \mathcal{S C}_{m}$ and $\ell \in \Gamma_{i}^{*}$, we have

$$
f_{\ell}(t)=\sum_{k=2}^{\infty} \sum_{p \in G_{k, \ell}^{1}} \xi(k, \ell, p)^{\alpha_{m}} f_{c(k, \ell, p)}(t+\ln \xi(k, \ell, p))+z_{\ell}^{\left(\alpha_{m}\right)}(t),
$$

where $z_{\ell}^{\left(\alpha_{m}\right)}(t)$ is obtained from that in (4.18) by replacing $n$ with $n_{t}$. For $m=1, \ldots, \eta$, $i \in \mathcal{S C}_{m}, \ell \in \Gamma_{i}$, let $\mu_{\ell^{\prime} \ell}^{\left(\alpha_{m}\right)}$ be the discrete measure such that for $2 \leq k \leq \varkappa_{\ell}, p \in G_{k, \ell}^{1}$, $\ell^{\prime}=c(k, \ell, p) \in \Gamma_{j}$ and $j \in J_{\ell}$,

$$
\mu_{\ell \ell^{\prime}}^{\left(\alpha_{m}\right)}(-\ln \xi(k, \ell, p)):=\xi(k, \ell, p)^{\alpha_{m}}
$$

Then (see (1.10))

$$
\mu_{\ell \ell^{\prime}}^{\left(\alpha_{m}\right)}(\mathbf{R})=\sum_{k=2}^{\varkappa_{\ell}} \sum_{p \in G_{k, \ell}^{1}} \xi(k, \ell, p)^{\alpha_{m}} \text { and } F_{\ell}\left(\alpha_{m}\right)=\sum_{\ell^{\prime} \in \Gamma} \sum_{k=2}^{\varkappa_{\ell}} \sum_{p \in G_{k, \ell}^{1}} \xi(k, \ell, p)^{\alpha_{m}}
$$

We summarize the above derivations in the following theorem.

Theorem 4.3. Let $\mu=\sum_{i=1}^{q} \mu_{i}$ be a graph-directed self-similar measure on $\mathbf{R}$. Assume that $\mu$ satisfies (EFT) with $\left\{\Omega_{i}\right\}_{i=1}^{q}$ being an EFT-family and assume that there exists a regular basic pair. Let $\Omega=\bigcup_{i=1}^{q} \Omega_{i}$ and $\Delta_{\mu}$ be defined on $\Omega$. Let $\mathbf{f}, \mathbf{M}_{\boldsymbol{\alpha}}$ and $\mathbf{z}$ be defined as in (1.8). Then $\mathbf{f}$ satisfies the vector-valued renewal equation $\mathbf{f}=\mathbf{f} * \mathbf{M}_{\boldsymbol{\alpha}}+\mathbf{z}$.

\subsection{Proofs of Theorems 1.1 and 1.6}

We first prove a result relating strong connectedness of a graph and irreducibility of the corresponding matrix.

Proposition 4.4. Let $\mu=\sum_{i=1}^{q} \mu_{i}$ be a graph-self-similar measure defined by a GIFS $G=(V, E)$ on $\mathbf{R}$. Assume that $\mu$ satisfies $(E F T)$ with $\left\{\Omega_{i}\right\}_{i=1}^{q}$ being an EFT-family and assume that there exists a regular basic pair $(\mathbf{B}, \mathbf{P}):=\left(\left\{B_{1, \ell}\right.\right.$, $\left.\left.\left\{\mathbf{P}_{k, \ell}\right\}_{k \geq 1}\right\}\right)_{\ell \in \Gamma}$. Let $\Omega=\bigcup_{i=1}^{q} \Omega_{i}, \Delta_{\mu}$ be the Dirichlet Laplacian defined by $\mu$, and let $\mathbf{M}(\boldsymbol{\alpha} ; \infty)$ be defined as in (1.9). Then $\mathbf{M}(\boldsymbol{\alpha} ; \infty)$ is irreducible if and only if $G$ is strongly connected. 
Proof. Assume that $G$ has $\eta$ strongly connected components, and $\mathbf{M}(\boldsymbol{\alpha} ; \infty)$ is irreducible. Then for any $\ell, \ell^{\prime} \in \Gamma$, there exists a path $\gamma=\left(\ell_{1}, \ldots, \ell_{n}\right)$ from $\ell_{1}$ to $\ell_{n}$ satisfying $\ell_{1}=\ell$ and $\ell_{n}=\ell^{\prime}$ such that

$$
\mu_{\gamma}=\mu_{\ell_{1} \ell_{2}}^{\left(\alpha_{m_{1}}\right)} * \ldots * \mu_{\ell_{n-1} \ell_{n}}^{\left(\alpha_{m_{n-1}}\right)} \neq 0
$$

where $\ell_{j} \in \Gamma_{i_{j}}, i_{j} \in \mathcal{S C}_{m_{j}}$ and $m_{j} \in\{1, \ldots, \eta\}$ for $j=1, \ldots, n$. It means that

$$
\mu_{\ell_{j} \ell_{j+1}}^{\left(\alpha_{m_{j}}\right)} \neq 0 \quad \text { for } j=1, \ldots, n-1 \text {. }
$$

Thus there exists at least one $B_{j} \in \mathbf{P}_{k, \ell_{j}}^{1}, k \geq 2$, such that $B_{j}$ is $\mu$-equivalent to $B_{1, \ell_{j}}$. By the definition of $\mu$-equivalence, we see that there exists $e_{j} \in E^{i_{j}, i_{j+1}}$ such that $S_{e_{j}}\left(B_{1, \ell_{j+1}}\right)=B_{j}$, where $B_{1, \ell_{j+1}} \subseteq \Omega_{i_{j+1}}$ and $B_{j} \subseteq \Omega_{i_{j}}$. Hence $\mathbf{e}=e_{1} \ldots e_{n-1} \in E^{i_{1}, i_{n}}$, i.e., there exists a directed $\boldsymbol{e}$-path from $i_{1}$ to $i_{n}$, where $\ell \in \Gamma_{i_{1}}$ and $\ell^{\prime} \in \Gamma_{i_{n}}$. Hence, for any $\gamma$-path, there exists a corresponding directed $\boldsymbol{e}$-path.

Let $i, j \in V$.

(1) If $\Gamma_{i} \neq \varnothing$ and $\Gamma_{j} \neq \varnothing$, then for any $\ell \in \Gamma_{i}$ and any $\ell^{\prime} \in \Gamma_{j}$, there exists a corresponding directed $\boldsymbol{e}$-path from $i$ to $j$. Hence $G$ is strongly connected.

(2) If $\Gamma_{i}=\varnothing$ and $\Gamma_{j} \neq \varnothing$, then there exists some $\ell_{1} \in \Gamma$ such that $B_{1, \ell_{1}}$ is $\mu$-equivalent to $\Omega_{i}$. Assume that $\ell_{1} \in \Gamma_{i_{1}}$ for $i_{1} \in V$. Then we have $B_{1, \ell_{1}} \subseteq \Omega_{i_{1}}$. By the definition of $\mu$-equivalence, there exists some $\boldsymbol{e}_{1} \in E^{i_{1}, i}$ such that $S_{\boldsymbol{e}_{1}}\left(\Omega_{i}\right)=B_{1, \ell}$. Since $\Gamma_{i_{1}} \neq \varnothing$ and $\Gamma_{j} \neq \varnothing$, there exists a corresponding directed $\boldsymbol{e}$-path from $i_{1}$ to $j$. Thus there exists a $\boldsymbol{e}$-path from $i$ to $j$, and hence $G$ is strongly connected.

(3) If $\Gamma_{i}=\varnothing$ and $\Gamma_{j}=\varnothing$, then $\Omega_{i}$ and $\Omega_{j}$ are $\mu$-equivalent, and so there exists a directed $\boldsymbol{e}$-path from $i$ to $j$. Hence $G$ is strongly connected.

Conversely, assume that $G$ is strongly connected. Let $\ell \in \Gamma_{i}$ and $\ell^{\prime} \in \Gamma_{i^{\prime}}$.

(1) If there exists some $e \in E^{i, i^{\prime}}$ satisfying $S_{e}\left(\Omega_{i^{\prime}}\right) \subseteq B_{1, \ell} \subseteq \Omega_{i}$, then by the definition of (EFT), there exists some $B_{1} \in \mathbf{P}_{k, \ell}^{1}, k \geq 2$, such that $B_{1} \neq B_{1, \ell}$. It follows from (2.6) that $B_{1}$ is $\mu$-equivalent to some $B_{1, \ell^{\prime}}$ for $\ell^{\prime} \in \Gamma_{i^{\prime}}$. Hence, there exists a path $\gamma=\left(\ell, \ell^{\prime}\right)$ satisfying $\ell \in \Gamma_{i}$ and $\ell^{\prime} \in \Gamma_{i^{\prime}}$.

(2) If there exists some $e \in E^{i, i^{\prime}}$ satisfying $S_{e}\left(\Omega_{i^{\prime}}\right) \subseteq \Omega_{i}$, but $S_{e}\left(\Omega_{j}\right) \cap B_{1, \ell}=\varnothing$, then the strong connectedness of $G$ implies that there exists some $e_{1} \in E^{i, i_{1}}$ such that $S_{e_{1}}\left(\Omega_{i_{1}}\right) \subseteq B_{1, \ell} \subseteq \Omega_{i}$. By the definition of (EFT), there exists some $B_{1} \in \mathbf{P}_{k, \ell}^{1}$, $k \geq 2$, such that $B_{1} \neq B_{1, \ell}$. Using (2.6), we see that $B_{1}$ is $\mu$-equivalent to some $B_{1, \ell_{1}}$ for $\ell_{1} \in \Gamma_{i_{1}}$. Hence there exists a path $\gamma=\left(\ell, \ell_{1}\right)$ from $\ell$ to $\ell_{1}$. If there exists some $e_{2} \in E^{i_{1}, j}$ such that $S_{e_{2}}\left(\Omega_{j}\right) \subseteq B_{1, \ell_{1}} \subseteq \Omega_{i_{1}}$, then there exists a path $\gamma=\left(\ell_{1}, \ell^{\prime}\right)$. This is similar to (1). Hence there exists a path $\gamma=\left(\ell, \ell_{1}, \ell^{\prime}\right)$ from $\ell$ to $\ell^{\prime}$. If there exists some $e_{2} \in E^{i_{1}, i^{\prime}}$ satisfying $S_{e_{2}}\left(\Omega_{i^{\prime}}\right) \subseteq \Omega_{i_{1}}$, but $S_{e_{2}}\left(\Omega_{j}\right) \cap B_{1, \ell_{1}}=\varnothing$, then we continue. Since $G$ is strongly connected, we can obtain some $e_{j} \in E^{i_{j-1}, i_{j}}$ satisfying $S_{e_{j}}\left(\Omega_{i_{j}}\right) \subseteq$ $B_{1, \ell_{j-1}} \subseteq \Omega_{i_{j-1}}$. Hence there exists some $B_{j} \in \mathbf{P}_{k, \ell_{j-1}}$ such that $B_{j} \neq B_{1, \ell_{j-1}}$. The 
definition of (EFT) implies that there exists some $B_{j}$ that is $\mu$-equivalent to some $B_{1, \ell_{j}}$ for $\ell_{j} \in \Gamma_{i_{j}}$. Hence there exists a path $\gamma$ from $\ell$ to $\ell^{\prime}$.

Proof of Theorem 1.1. (a) It follows from (4.14) and Proposition 4.2 that $F_{\ell}(\alpha)$ is a positive continuous function of $\alpha$ for each $\ell \in \Gamma$. Combining this with the facts that $\lim _{\alpha \rightarrow \infty} F_{\ell}(\alpha)=0$ and $\lim _{\alpha \rightarrow \widetilde{\alpha}_{\ell}^{+}} F_{\ell}(\alpha)>1$, we obtain a unique $\alpha$ such that the spectral radius of $\mathbf{M}(\boldsymbol{\alpha} ; \infty)$ is 1 , where $\boldsymbol{\alpha}=(\alpha)$ consists of only one component.

(b) Let $\alpha$ be the unique number in part (a). Let $\mathbf{m}:=\left[m_{\ell \ell^{\prime}}^{(\alpha)}\right]=\left[\int_{0}^{\infty} t d \mu_{\ell \ell^{\prime}}^{(\alpha)}\right]$ be the moment matrix. Following the proof of [17, Theorem 1.1(b)], we need to show that some moment condition holds, and it suffices to show that $0<\sum_{\ell^{\prime} \in \Gamma} m_{\ell \ell^{\prime}}^{(\alpha)}<\infty$. It is easy to check that for $\ell \in \Gamma, \sum_{\ell^{\prime} \in \Gamma} m_{\ell \ell^{\prime}}^{(\alpha)}$ takes the following values:

$$
\sum_{\ell^{\prime} \in \Gamma} \sum_{k=2}^{\varkappa_{\ell}} \sum_{p \in G_{k, \ell}^{1}} \xi(k, \ell, p)^{\alpha}|\ln (\xi(k, \ell, p))| .
$$

It follows from $\lim _{\alpha \rightarrow \widetilde{\alpha}_{\ell}^{+}} F_{\ell}(\alpha)>1$ that there exists $\varepsilon>0$ such that $0<F_{\ell}(\alpha-\varepsilon)<\infty$. Thus

$$
\begin{aligned}
0 & <\sum_{\ell^{\prime} \in \Gamma} \sum_{k=2}^{\varkappa_{\ell}} \sum_{p \in G_{k, \ell}^{1}} \xi(k, \ell, p)^{\alpha}|\ln (\xi(k, \ell, p))| \\
& =\sum_{\ell^{\prime} \in \Gamma} \sum_{k=2}^{\varkappa_{\ell}} \sum_{p \in G_{k, \ell}^{1}} \xi(k, \ell, p)^{\alpha-\varepsilon} \xi(k, \ell, p)^{\varepsilon}|\ln (\xi(k, \ell, p))|<\infty .
\end{aligned}
$$

The last inequality follows from the fact $\lim _{t \rightarrow 0^{+}} t^{\varepsilon} \ln t=0$. By (4.13), we have

$$
\sum_{\ell^{\prime} \in \Gamma} \mu_{\ell \ell^{\prime}}^{(\alpha)}(0)=0<\sum_{\ell^{\prime} \in \Gamma} \mu_{\ell \ell^{\prime}}^{(\alpha)}(\infty),
$$

i.e., each column of $\mathbf{M}_{\boldsymbol{\alpha}}$ is nondegenerate at 0 . From Theorem $4.3, \mathbf{f}=\mathbf{f} * \mathbf{M}_{\boldsymbol{\alpha}}+\mathbf{z}$, where, by assumption, $\mathbf{z}$ is directly Riemann integrable on $\mathbf{R}$.

Proposition 4.4 and the fact that $G$ is strongly connected imply that $\mathbf{M}(\boldsymbol{\alpha} ; \infty)$ is irreducible. It follows from the above observations and [17, Theorem 4.1] that there exist positive constants $C_{1}$ and $C_{2}$ such that $0<C_{1} \leq \underline{\lim }_{t \rightarrow \infty} f_{\ell}(t) \leq \overline{\lim }_{t \rightarrow \infty} f_{\ell}(t) \leq$ $C_{2}<\infty$ for all $\ell \in \Gamma$. The definition in (4.7) implies that $C_{1} \leq \lambda^{-\alpha} N\left(\lambda,-\Delta_{\left.\mu_{i}\right|_{B_{1, \ell}}}\right) \leq$ $C_{2}$, which, together with (4.2), yields $C_{1} \lambda^{\alpha} \leq N\left(\lambda,-\Delta_{\left.\mu\right|_{\Omega}}\right) \leq C_{2} \lambda^{\alpha}$. Combining this, part (a), and the definition of $d_{s}$, we get $d_{s}=2 \alpha$.

(c) The assertion follows from Corollary A.2 and Theorem A.1 in the Appendix. This completes the proof. 
Proof of Theorem 1.6. (a) Proposition 4.2 and (4.22) imply that for $m=1, \ldots, \eta$, $i \in \mathcal{S C}_{m}$ and $\ell \in \Gamma_{i}, F_{\ell}\left(\alpha_{m}\right)$ is a positive continuous function of $\alpha_{m}$. Combining this with the assumptions that $\lim _{\alpha_{m} \rightarrow \infty} F_{\ell}\left(\alpha_{m}\right)=0$ and $\lim _{\alpha_{m} \rightarrow \widetilde{\alpha}_{\ell}^{+}} F_{\ell}\left(\alpha_{m}\right)>1$, we obtain a unique nonnegative vector $\boldsymbol{\alpha}=\left(\alpha_{1}, \ldots, \alpha_{\eta}\right)$ such that the spectral radii of $\mathbf{M}(\boldsymbol{\alpha} ; \infty)$ and all the other classes are equal to 1 .

(b) The proof is similar to that of Theorem 1.1(b). Since $G$ is not strongly connected, it follows from Proposition 4.4 that $\mathbf{M}(\boldsymbol{\alpha} ; \infty)$ is reducible. Let $\alpha:=$ $\max \left\{\alpha_{i}: i=1, \ldots, \eta\right\}$. As in the proof of [17, Theorem 1.1(b), Case 2], we have

$$
\lim _{t \rightarrow \infty} f_{\ell}^{(\beta)}(t)=0 \quad \text { for all } \ell \in \Gamma_{i}, i \in\{1, \ldots, q\} \text { and all } \beta<\alpha .
$$

Moreover, there exists some $\ell_{0} \in \Gamma_{i}$ such that $\underline{\lim }_{t \rightarrow \infty} f_{\ell_{0}}^{(\alpha)}(t)>0$. The definition of $f_{\ell}(t)$ implies that $N\left(\lambda,-\Delta_{\left.\mu_{i}\right|_{B_{1, \ell}}}\right)=o\left(\lambda^{\beta}\right)$ for $\overline{\ell \in \Gamma_{i}}$ and $\beta<\alpha$ and $\underline{\lim }_{\lambda \rightarrow \infty} \lambda^{-\alpha} N(\lambda$, $\left.-\Delta_{\left.\mu_{i}\right|_{B_{1, \ell_{0}}}}\right)>0$. Thus $N\left(\lambda,-\Delta_{\mu}\right)=o\left(\lambda^{\beta}\right)$ for any $\beta<\alpha$ and $\underline{\lim }_{\lambda \rightarrow \infty} \lambda^{-\alpha} N(\lambda$, $\left.-\Delta_{\mu}\right)>0$. Hence $\bar{d}_{s}\left(-\Delta_{\mu}\right) \leq 2 \alpha$ and $\underline{d}_{s}\left(-\Delta_{\mu}\right) \geq 2 \alpha$, which completes the proof.

(c) The proof is similar to that of Theorem 1.1(c).

\section{Strongly connected GIFSs on $\mathbf{R}$}

In this section, we compute the spectral dimension of some graph-directed selfsimilar measures defined by a strongly connected GIFS $G=(V, E)$ that has overlaps.

\subsection{A strongly connected GIFS}

We first show that the graph-directed self-similar measures defined by the strongly connected GIFS in Example 1.2 satisfy (EFT).

Proposition 5.1. Let $\mu=\mu_{1}+\mu_{2}$ be a graph-directed self-similar measure defined by a $G I F S G=(V, E)$ in Example 1.2 together with a probability matrix $\left(p_{e}\right)_{e \in E}$. Then $\mu$ satisfies $(E F T)$ with $\left\{\Omega_{1}, \Omega_{2}\right\}=\{(0,1),(0,1)\}$ being an EFT-family and there exists a regular basic pair.

To prove Proposition 5.1, we first summarize some elementary properties. Throughout this subsection, we let $\Omega_{1}=\Omega_{2}:=(0,1)$ and $\zeta_{1}:=1-r$. Define

$$
\begin{array}{ll}
B_{1,1}:=S_{e_{1}}\left(\Omega_{1}\right) \cup S_{e_{2}}\left(\Omega_{2}\right)=\left(0, r+\rho \zeta_{1}\right), & B_{1,2}:=S_{e_{3}}\left(\Omega_{1}\right)=\left(\zeta_{1}, 1\right), \\
B_{1,3}:=S_{e_{5}}\left(\Omega_{1}\right)=(0, \rho), & B_{1,4}:=S_{e_{4}}\left(\Omega_{2}\right)=\left(\zeta_{1}, 1\right) .
\end{array}
$$

Using (2.3) and (2.4), we see that

$$
p_{e_{1}}+p_{e_{2}}+p_{e_{3}}=1, \quad p_{e_{4}}+p_{e_{5}}=1 .
$$


and

$$
\mu_{1}=p_{e_{1}} \mu_{1} \circ S_{e_{1}}^{-1}+p_{e_{2}} \mu_{2} \circ S_{e_{2}}^{-1}+p_{e_{3}} \mu_{1} \circ S_{e_{3}}^{-1}, \quad \mu_{2}=p_{e_{4}} \mu_{2} \circ S_{e_{4}}^{-1}+p_{e_{5}} \mu_{1} \circ S_{e_{5}}^{-1} .
$$

Moreover $\mu=\mu_{1}+\mu_{2}$.

Lemma 5.2. Let $\left\{S_{e_{i}}\right\}_{i=1}^{5}$ be as in (1.12). Then $S_{e_{1} e_{3}}=S_{e_{2} e_{5}}$.

Lemma 5.3. Assume the hypotheses of Proposition 5.1. Let $B_{1, \ell}, \ell=1,2,3,4$, be defined as in (5.1). Then

(a) $\left.\mu_{1}\right|_{S_{e_{1}}\left(B_{1,1}\right)}=\left.p_{e_{1}} \mu_{1}\right|_{B_{1,1}} \circ S_{e_{1}}^{-1}$;

(b) $\left.\mu_{1}\right|_{S_{e_{1}}\left(B_{1,2}\right)}=\left.\left(p_{e_{1} e_{3}}+p_{e_{2} e_{5}}\right) p_{e_{5}}^{-1} \cdot \mu_{2}\right|_{B_{1,3}} \circ\left(S_{e_{1} e_{3}} S_{e_{5}}^{-1}\right)^{-1}$;

(c) $\left.\mu_{1}\right|_{S_{e_{2}}\left(B_{1,4}\right)}=\left.p_{e_{2}} \mu_{2}\right|_{B_{1,4}} \circ S_{e_{2}}^{-1}$;

(d) for $\ell=1,2,\left.\mu_{1}\right|_{S_{e_{3}}\left(B_{1, \ell}\right)}=\left.p_{e_{3}} \mu_{1}\right|_{B_{1, \ell}} \circ S_{e_{3}}^{-1}$;

(e) for $\ell=3,4,\left.\mu_{2}\right|_{S_{e_{4}}\left(B_{1, \ell}\right)}=\left.p_{e_{4}} \mu_{2}\right|_{B_{1, \ell}} \circ S_{e_{4}}^{-1}$;

(f) for $\ell=1,2$ and $k \geq 0,\left.\mu_{2}\right|_{S_{e_{5} e_{3}^{k}}\left(B_{1, \ell}\right)}=\left.p_{e_{5} e_{3}^{k}} \mu_{1}\right|_{B_{1, \ell}} \circ S_{e_{5} e_{3}^{k}}^{-1}$.

Proof. (a) It follows from (1.12) and (5.1) that $S_{e_{1}}\left(\Omega_{1}\right)=(0, \rho), S_{e_{2}}\left(\Omega_{2}\right)=\left(\rho \zeta_{1}\right.$, $\left.r+\rho \zeta_{1}\right)$ and $S_{e_{1}}\left(B_{1,1}\right)=\left(0, \rho r+\rho^{2} \zeta_{1}\right)$. Since $\rho+2 r-\rho r \leq 1$, we have $\rho r+\rho^{2} \zeta_{1} \leq \rho \zeta_{1}$, and hence $S_{e_{1}}\left(B_{1,1}\right) \subseteq S_{e_{1}}\left(\Omega_{1}\right) \backslash S_{e_{2}}\left(\Omega_{2}\right)$. So for any $A \subseteq S_{e_{1}}\left(B_{1,1}\right)$, i.e., $S_{e_{1}}^{-1}(A) \subseteq$ $B_{1,1}$, we get $\mu_{1}(A)=\left.p_{e_{1}} \mu_{1}\right|_{B_{1,1}} \circ S_{e_{1}}^{-1}(A)$.

(b) By Lemma 5.2 we have $S_{e_{1} e_{3}}\left(\Omega_{1}\right)=S_{e_{2} e_{5}}\left(\Omega_{1}\right)$. Then for any $A \subseteq S_{e_{1}}\left(B_{1,2}\right)$, $S_{e_{1} e_{3}}^{-1}(A)=S_{e_{2} e_{5}}^{-1}(A) \subseteq \Omega_{1}$, and hence

$$
\mu_{1}(A)=\left.\left(p_{e_{1} e_{3}}+p_{e_{2} e_{5}}\right) \mu_{1}\right|_{\Omega_{1}} \circ S_{e_{1} e_{3}}^{-1}(A) .
$$

For any $B \subseteq B_{1,3}, S_{e_{5}}^{-1}(B) \subseteq \Omega_{1}$, so $\mu_{2}(B)=\left.p_{e_{5}} \mu_{1}\right|_{\Omega_{1}} \circ S_{e_{5}}^{-1}(B)$, and hence

$$
\left.\mu_{1}\right|_{\Omega_{1}}=\left.p_{e_{5}}^{-1} \mu_{2}\right|_{B_{1,3}} \circ S_{e_{5}} .
$$

Combining this with (5.4), we have $\left.\mu_{1}\right|_{S_{e_{1}}\left(B_{1,2}\right)}=\left.\left(p_{e_{1} e_{3}}+p_{e_{2} e_{5}}\right) p_{e_{5}}^{-1} \mu_{2}\right|_{B_{1,3}} \circ S_{e_{5}} S_{e_{1} e_{3}}^{-1}$.

(c) Using (1.12) and (5.1), we get $S_{e_{2}}\left(B_{1,4}\right)=\left((\rho+r) \zeta_{1}, r+\rho \zeta_{1}\right)$. Since $\rho<(\rho+$ $r) \zeta_{1}, S_{e_{2}}\left(B_{1,4}\right) \subseteq S_{e_{2}}\left(\Omega_{2}\right) \backslash S_{e_{1}}\left(\Omega_{1}\right)$, and hence $\mu_{1}\left|(A)=p_{e_{2}} \mu_{2}\right|_{B_{1,4}} \circ S_{e_{2}}^{-1}(A)$ for any $A \subseteq S_{e_{2}}\left(B_{1,4}\right)$.

(d) and (e) follow from (5.3) and the facts $S_{e_{3}}\left(B_{1, \ell}\right) \subseteq S_{e_{3}}\left(\Omega_{1}\right), S_{e_{4}}\left(B_{1, \ell^{\prime}}\right) \subseteq$ $S_{e_{4}}\left(\Omega_{2}\right)$ for $\ell=1,2$ and $\ell^{\prime}=3,4$.

(f) First, we show that for $k \geq 1$ and $\ell=1,2$,

$$
\left.\mu_{1}\right|_{S_{3}^{k}\left(B_{1, \ell}\right)}=\left.p_{e_{3}^{k}} \mu_{1}\right|_{B_{1, \ell}} \circ S_{e_{3}^{k}}^{-1} .
$$

It follows from (d) that (5.6) holds for $k=1$. Assume that (5.6) holds for $k=m$, i.e., $\left.\mu_{1}\right|_{S_{e_{3}^{m}}\left(B_{1, \ell}\right)}=\left.p_{e_{3}^{m}} \mu_{1}\right|_{B_{1, \ell}} \circ S_{e_{3}^{m}}^{-1}$. For $k=m+1$, note that $S_{e_{3}^{m}}\left(B_{1, \ell}\right) \subseteq B_{1,2}$ for $m \geq 1$. Combining this with $(\mathrm{d})$, we have

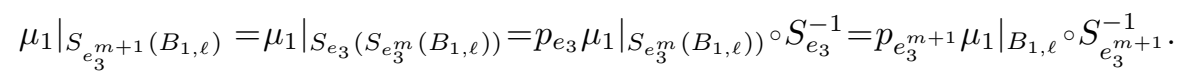



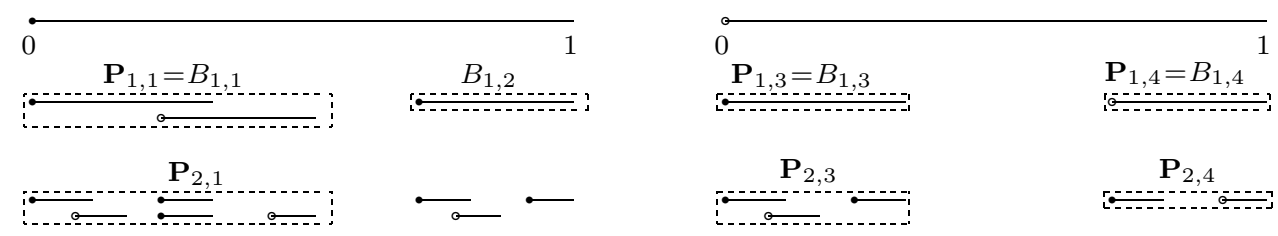

Figure 2. $\mu$-partitions $\mathbf{P}_{k, \ell}$ of $B_{1, \ell}$ for the GIFS defined in (1.12). The figure is drawn with $\rho=1 / 3$ and $r=2 / 7$.

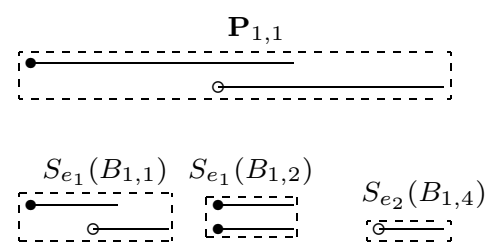

Figure 3. $\mu$-partitions $\mathbf{P}_{2,1}$.

It is obvious that $S_{e_{5}}\left(B_{1, \ell}\right) \subseteq S_{e_{5}}\left(\Omega_{1}\right)$ for $\ell=1,2$. Hence

$$
\left.\mu_{2}\right|_{S_{e_{5}}\left(B_{1, \ell}\right)}=\left.p_{e_{5}} \mu_{1}\right|_{B_{1, \ell}} \circ S_{e_{5}}^{-1} \quad \text { for } \ell=1,2 .
$$

Thus (f) holds for $k=0$. Note that $S_{e_{3}^{m}}\left(B_{1, \ell}\right) \subseteq B_{1,2}$ for $m \geq 1$. Applying (5.7) followed by (5.6), we have for $k \geq 1$,

$$
\left.\mu_{2}\right|_{S_{e_{5} e_{3}^{k}}\left(B_{1, \ell}\right)}=\left.\mu_{2}\right|_{S_{e_{5}}\left(S_{e_{3}}^{k}\left(B_{1, \ell}\right)\right)}=\left.p_{e_{5}} \mu_{1}\right|_{S_{e_{3}^{k}}\left(B_{1, \ell}\right)} \circ S_{e_{5}}^{-1}=\left.p_{e_{5} e_{3}^{k}} \mu_{1}\right|_{B_{1, \ell}} \circ S_{e_{5} e_{3}^{k}}^{-1}
$$

This completes the proof.

Proof of Proposition 5.1. Note that $\left\{\Omega_{1}, \Omega_{2}\right\}$ is invariant under $G=(V, E)$. It is obvious that any two elements of $\left\{B_{1,1}, B_{1,3}, B_{1,4}\right\}$ are measure disjoint. Let $\Gamma:=\{1,3,4\}$ and $\mathbf{B}:=\left\{B_{1, \ell}: \ell \in \Gamma\right\}$. Define $\mathbf{P}_{1, \ell}:=\left\{B_{1, \ell}\right\}$ for $\ell \in \Gamma$. If for some $k \geq 2$ and $\ell \in \Gamma, \mathbf{P}_{k, \ell}$ (see Figure 2 ) is a well-defined $\mu$-partition of $B_{1, \ell}$, then we let $\mathbf{P}_{k, \ell}^{1}$ and $\mathbf{P}_{k, \ell}^{2}$ be defined as in (2.6) with respect to $\mathbf{B}$.

For $\ell=1$, define $\mathbf{P}_{2,1}:=\left\{S_{e_{1}}\left(B_{1,1}\right), S_{e_{1}}\left(B_{1,2}\right), S_{e_{2}}\left(B_{1,4}\right)\right\}$ (see Figure 3 ). Note that any two elements of $\mathbf{P}_{2,1}$ are measure disjoint. Thus $\mathbf{P}_{2,1}$ is a refining $\mu$-partition of $B_{1,1}$. It follows from Lemma $5.3(\mathrm{a}, \mathrm{b}, \mathrm{c})$ that

$$
\mathbf{P}_{2,1}^{1}=\left\{S_{e_{1}}\left(B_{1,1}\right), S_{e_{1}}\left(B_{1,2}\right), S_{e_{2}}\left(B_{1,4}\right)\right\} \quad \text { and } \quad \mathbf{P}_{2,1}^{2}=\varnothing .
$$

Hence condition (1) of Definition 2.1 holds for $\ell=1$. For $k \geq 3$, define $\mathbf{P}_{k, 1}=\mathbf{P}_{2,1}$. Then conditions (2) and (3) of Definition 2.1 hold for $\ell=1$.

For $\ell=3$, define $\mathbf{P}_{2,3}=\left\{S_{e_{5}}\left(B_{1,1}\right), S_{e_{5}}\left(B_{1,2}\right)\right\}$ (see Figure 4 ). It is easy to see that $S_{e_{5}}\left(B_{1,1}\right), S_{e_{5}}\left(B_{1,2}\right)$ are measure disjoint, and $S_{e_{5}}\left(B_{1, i}\right) \subseteq B_{1,3}$ for $i=1,2$. Thus 

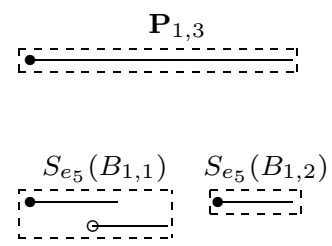

Figure 4. $\mu$-partitions $\mathbf{P}_{2,3}$ and $\mathbf{P}_{2,4}$.
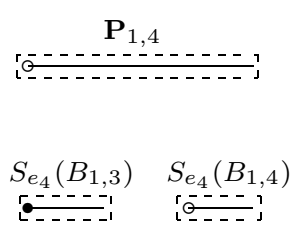

0

$S_{e_{1}}\left(\Omega_{1}\right)-S_{e_{2}}\left(\Omega_{1}\right)$
10

$S_{e_{3}}\left(\Omega_{1}\right)$
$0 \quad \Omega_{2}$

$S_{e_{5}}\left(\Omega_{1}\right)$

Figure 5. First iteration of the GIFS defined in (1.15), where $\Omega_{1}=\Omega_{2}=(0,1)$. The figure is drawn with $\rho=1 / 3$ and $r=2 / 7$.

$\mathbf{P}_{2,3}$ is a refining $\mu$-partition of $B_{1,3}$. By Lemma 5.3(f), we have $\mathbf{P}_{2,3}^{1}=\left\{S_{e_{5}}\left(B_{1,1}\right)\right\}$ and $\mathbf{P}_{2,3}^{2}=\left\{S_{e_{5}}\left(B_{1,2}\right)\right\}$. Hence condition (1) of Definition 2.1 holds for $\ell=3$. For $k \geq$ 3 , define $\mathbf{P}_{k, 3}=\mathbf{P}_{k-1,3}^{1} \cup\left\{S_{e_{5} e_{3}^{k-2}}\left(B_{1,1}\right), S_{e_{5} e_{3}^{k-2}}\left(B_{1,2}\right)\right\}$, Lemma 5.3(f) implies that $\mathbf{P}_{k, 3}^{1}=\mathbf{P}_{k-1,3}^{1} \cup\left\{S_{e_{5} e_{3}^{k-2}}\left(B_{1,1}\right)\right\}$ and $\mathbf{P}_{k, 3}^{2}=\left\{S_{e_{5} e_{3}^{k-2}}\left(B_{1,2}\right)\right\}$. Consequently, condition (2) of Definition 2.1 holds for $\ell=3$. Since the closure of $S_{e_{5} e_{3}^{k-2}}\left(B_{1,2}\right)$ converges to a point as $k \rightarrow \infty$, we get $\lim _{k \rightarrow \infty} \mu\left(S_{e_{5} e_{3}^{k-2}}\left(B_{1,2}\right)\right)=0$. We conclude that condition (3) of Definition 2.1 holds.

For $\ell=4$, define $\mathbf{P}_{2,4}:=\left\{S_{e_{4}}\left(B_{1,3}\right), S_{e_{4}}\left(B_{1,4}\right)\right\}$ (see Figure 4). Note that $S_{e_{4}}\left(B_{1,3}\right), S_{e_{4}}\left(B_{1,4}\right)$ are measure disjoint, and $S_{e_{4}}\left(B_{1, i}\right) \subseteq B_{1,4}$ for $i=3,4$. Thus $\mathbf{P}_{2,4}$ is a refining $\mu$-partition of $B_{1,4}$. Lemma 5.3(e) implies that $\mathbf{P}_{2,4}^{1}=\mathbf{P}_{2,4}$ and $\mathbf{P}_{2,4}^{2}=\varnothing$. Hence condition (1) of Definition 2.1 holds for $\ell=4$. For $k>2$, define $\mathbf{P}_{k, 4}:=\mathbf{P}_{2,4}$. It follows that conditions (2) and (3) of Definition 2.1 hold for $\ell=4$. Hence the first assertion follows. Finally, the regularity of $\left(\left\{B_{1, \ell}\right\},\left\{\mathbf{P}_{k, \ell}\right\}_{k \geq 1}\right)_{\ell \in \Gamma}$ is obvious.

\subsection{Spectral dimension of $\mu$ in Proposition 5.1}

In this subsection, we derive the vector-valued renewal equations and compute the spectral dimension of $\mu$ defined by the strongly connected GIFS in Proposition 5.1.

Let $\left\{S_{e_{i}}\right\}_{i=1}^{5}$ be a GIFS in (1.12), $\left(p_{e}\right)_{e \in E}$ be a probability matrix, and $\mu$ be the associated graph-directed self-similar measure. For $\ell \in \Gamma:=\{1,3,4\}$ and $k \geq 1$, let $B_{1, \ell}$ be defined as in (5.1), and $\mathbf{P}_{k, \ell}$ be as in the proof of Proposition 5.1. Then $\mu$ satisfies (EFT) with $\left\{\Omega_{1}, \Omega_{2}\right\}=\{(0,1),(0,1)\}$ being an EFT-family, and $(\mathbf{B}, \mathbf{P}):=\left(\left\{B_{1, \ell}\right\},\left\{\mathbf{P}_{k, \ell}\right\}_{k \geq 1}\right)_{\ell \in \Gamma}$ being a regular basic pair. 
In the rest of this subsection, we use the notation defined in Section 4.1. For $\ell \in \Gamma, i=1,2$ and $k \geq 2$, let $\mathbf{P}_{k, \ell}^{i}$ be defined as in (2.6). For $i=1,2$, let $\Gamma_{i}$ and $\mathbf{B}_{i}$ be defined as in (4.1). Since $B_{1,1} \subseteq \Omega_{1}$ and $B_{1, \ell} \subseteq \Omega_{2}$ for $\ell=3,4$, we have $\Gamma_{1}=\{1\}$, $\Gamma_{2}=\{3,4\}, \mathbf{B}_{1}=\left\{B_{1,1}\right\}$ and $\mathbf{B}_{2}=\left\{B_{1,3}, B_{1,4}\right\}$. Let

$$
\begin{array}{lll}
B_{2,1,1}=S_{e_{1}}\left(B_{1,1}\right), & B_{2,1,2}=S_{e_{1}}\left(B_{1,2}\right), & B_{2,1,3}=S_{e_{2}}\left(B_{1,4}\right), \\
B_{2,3,1}=S_{e_{5}}\left(B_{1,1}\right), & B_{2,3,2}=S_{e_{5}}\left(B_{1,2}\right), & B_{2,4,1}=S_{e_{4}}\left(B_{1,3}\right),
\end{array}
$$

Then

$$
\begin{array}{lll}
\mathbf{P}_{2,1}^{1}=\left\{B_{2,1,1}, B_{2,1,2}, B_{2,1,3}\right\}, & \mathbf{P}_{2,3}^{1}=\left\{B_{2,3,1}\right\}, & \mathbf{P}_{2,4}^{1}=\left\{B_{2,4,1}, B_{2,4,2}\right\}, \\
\mathbf{P}_{2,1}^{2}=\varnothing, & \mathbf{P}_{2,3}^{2}=\left\{B_{2,3,2}\right\}, & \mathbf{P}_{2,4}^{2}=\varnothing .
\end{array}
$$

Define $B_{k, 3,1}=\left\{S_{e_{5} e_{3}^{k-2}}\left(B_{1,1}\right)\right\}, B_{k, 3,2}=\left\{S_{e_{5} e_{3}^{k-2}}\left(B_{1,2}\right)\right\}$. Then $\mathbf{P}_{k, 3}^{1}=\left\{B_{k, 3,1}\right\}$ and $\mathbf{P}_{k, 3}^{2}=\left\{B_{k, 3,2}\right\}$. Hence $\Gamma_{1}^{\prime}=\{1\}, \Gamma_{1}^{*}=\varnothing, \Gamma_{2}^{\prime}=\{4\}, \Gamma_{2}^{*}=\{3\}, \varkappa_{1}=\varkappa_{4}=2, \varkappa_{3}=\infty$, and

$$
\begin{array}{ll}
G_{2,1}^{1}=\{1,2,3\}, & G_{2,1}^{2}=\varnothing, \\
G_{k, 3}^{1}=\{1\}, & G_{k, 3}^{2}=\{2\}, \text { for } k \geq 2, \\
G_{2,4}^{1}=\{1,2\}, & G_{2,4}^{2}=\varnothing .
\end{array}
$$

For $\ell \in \Gamma$, let $J_{\ell}$ be defined as in (4.3). Then $J_{1}=\{1,2\}, J_{3}=\{1\}, J_{4}=\{2\}$.

Lemma 5.4. Let $\xi(\cdot, \cdot, \cdot)$ and $c(\cdot, \cdot, \cdot)$ be defined as in Proposition 4.2. Then

(a) $\xi(2,1,1)=p_{e_{1}} \rho, c(2,1,1)=1$;

(b) $\xi(2,1,2)=\left(p_{e_{1} e_{3}}+p_{e_{2} e_{5}}\right) p_{e_{5}}^{-1} \cdot r, c(2,1,2)=3$;

(c) $\xi(2,1,3)=p_{e_{4}} r, c(2,1,3)=4$;

(d) for $k \geq 2, \xi(k, 3,1)=p_{e_{5} e_{3}^{k-2} \rho r^{k-2}}, c(k, 3,1)=1$;

(e) $\xi(2,4,1)=p_{e_{4}} r, c(2,4,1)=3$;

(f) $\xi(2,4,2)=p_{e_{4}} r, c(2,4,2)=4$.

Proof. (a)-(f) Lemma 5.3 implies that

$$
\begin{aligned}
& B_{1,1} \simeq_{\mu, S e_{1}, p_{e_{1}}} B_{2,1,1}, \quad B_{1,3} \simeq_{\mu, S_{e_{1} e_{3}}{ }^{\circ} S_{e_{5}}^{-1},\left(p_{e_{1} e_{3}+p_{e_{2} e_{5}}}\right) / p_{e_{5}}} B_{2,1,2},
\end{aligned}
$$

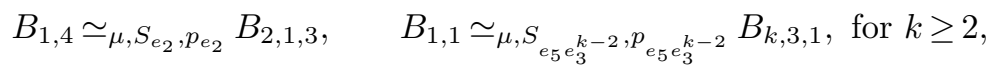

$$
\begin{aligned}
& B_{1,3} \simeq_{\mu, S_{e_{4}}, p_{e_{4}}} B_{2,4,1}, \quad B_{1,4} \simeq{ }_{\mu, S_{e_{4}}, p_{e_{4}}} B_{2,4,2} .
\end{aligned}
$$

The results follow. 
Using Lemma 5.4 and the discussions preceding it, we can express the vectorvalued renewal equations (4.8) and (4.12) precisely as

$$
\begin{aligned}
f_{1}(t)= & \left(p_{e_{1}} \rho\right)^{\alpha} f_{1}\left(t+\ln \left(p_{e_{1}} \rho\right)\right)+\left(\left(p_{e_{1} e_{3}}+p_{e_{2} e_{5}}\right) p_{e_{5}}^{-1} r\right)^{\alpha} f_{3}\left(t+\ln \left(\left(p_{e_{1} e_{3}}+p_{e_{2} e_{5}}\right) p_{e_{5}}^{-1} r\right)\right) \\
& +\left(p_{e_{2}} r\right)^{\alpha} f_{4}\left(t+\ln \left(p_{e_{2}} r\right)\right)+z_{1}^{(\alpha)}(t) \\
f_{3}(t)= & \sum_{k=0}^{\infty}\left(p_{e_{5} e_{3}^{k}} \rho r^{k}\right)^{\alpha} f_{1}\left(t+\ln \left(p_{e_{5} e_{3}^{k}} \rho r^{k}\right)\right)+z_{3}^{(\alpha)}(t) \\
f_{4}(t)= & \sum_{\ell=3}^{4}\left(p_{e_{4}} r\right)^{\alpha} f_{\ell}\left(t+\ln \left(p_{e_{4}} r\right)\right)+z_{4}^{(\alpha)}(t)
\end{aligned}
$$

where

$$
\begin{aligned}
& z_{1}^{(\alpha)}(t):=e^{-\alpha t} \varepsilon(2,1), \quad z_{4}^{(\alpha)}(t):=e^{-\alpha t} \varepsilon(2,4), \\
& z_{3}^{(\alpha)}(t):=e^{-\alpha t}\left(N\left(\lambda,-\Delta_{\left.\mu_{2}\right|_{B_{n_{t}, 3,2}}}\right)+\varepsilon\left(n_{t}, 3\right)\right) .
\end{aligned}
$$

For $\ell, \ell^{\prime} \in \Gamma$, let $\mu_{\ell \ell^{\prime}}^{(\alpha)}$ be the discrete measure defined as in (4.13). Then

$$
\begin{aligned}
& \mu_{11}^{(\alpha)}\left(-\ln \left(p_{e_{1}} \rho\right)\right)=\left(p_{e_{1}} \rho\right)^{\alpha}, \\
& \mu_{13}^{(\alpha)}\left(-\ln \left(\left(p_{e_{1} e_{3}}+p_{e_{2} e_{5}}\right) p_{e_{5}}^{-1} r\right)\right)=\left(\left(p_{e_{1} e_{3}}+p_{e_{2} e_{5}}\right) p_{e_{5}}^{-1} r\right)^{\alpha}, \\
& \mu_{14}^{(\alpha)}\left(-\ln \left(p_{e_{2}} r\right)\right)=\left(p_{e_{2}} r\right)^{\alpha}, \\
& \mu_{31}^{(\alpha)}\left(-\ln \left(p_{e_{5} e_{3}^{k}} \rho r^{k}\right)\right)=\left(p_{e_{5} e_{3}^{k}} \rho r^{k}\right)^{\alpha} \text { for } k=0,1, \ldots, \\
& \mu_{43}^{(\alpha)}\left(-\ln \left(p_{e_{4}} r\right)\right)=\mu_{44}^{(\alpha)}\left(-\ln \left(p_{e_{4}} r\right)\right)=\left(p_{e_{4}} r\right)^{\alpha} .
\end{aligned}
$$

Also,

$$
\mathbf{M}(\boldsymbol{\alpha} ; \infty)=\left(\begin{array}{ccc}
\left(p_{e_{1}} \rho\right)^{\alpha} & \left(\left(p_{e_{1} e_{3}}+p_{e_{2} e_{5}}\right) p_{e_{5}}^{-1} r\right)^{\alpha} & \left(p_{e_{2}} r\right)^{\alpha} \\
\left(\left(p_{e_{5}} \rho\right)^{\alpha}\right) /\left(1-\left(p_{e_{3}} r\right)^{\alpha}\right) & 0 & 0 \\
0 & \left(p_{e_{4}} r\right)^{\alpha} & \left(p_{e_{4}} r\right)^{\alpha}
\end{array}\right)
$$

Proposition 5.5. For $\ell=1,3,4$, let $F_{\ell}(\alpha)$ and $\tilde{\alpha}_{\ell}$ be defined as in (1.10). If $\left(p_{e_{1} e_{3}}+p_{e_{2} e_{5}}\right) p_{e_{5}}^{-1} r<1$, then $\lim _{\alpha \rightarrow \infty} F_{\ell}(\alpha)=0$, and $\tilde{\alpha}_{\ell}=0$ for $\ell=1,3,4$. Moreover, $F_{\ell}(0)>1$ for $\ell=1,4$, and $\lim _{\alpha \rightarrow 0^{+}} F_{3}(\alpha)=\infty$.

Proof. By the definition of $F_{\ell}(\alpha)$, we see that

$$
\begin{aligned}
& F_{1}(\alpha)=\left(p_{e_{1}} \rho\right)^{\alpha}+\left(\left(p_{e_{1} e_{3}}+p_{e_{2} e_{5}}\right) p_{e_{5}}^{-1} r\right)^{\alpha}+\left(p_{e_{2}} r\right)^{\alpha} \\
& F_{3}(\alpha)=\left(\left(p_{e_{5}} \rho\right)^{\alpha}\right) /\left(1-\left(p_{e_{3}} r\right)^{\alpha}\right)
\end{aligned}
$$

The assertions follow immediately, with $F_{1}(0)=3$ and $F_{4}(0)=2$. 
It follows from the Proposition 5.5 that there exists a unique $\alpha>0$ such that the spectral radius of $\mathbf{M}(\boldsymbol{\alpha} ; \infty)$ is 1 , where $\boldsymbol{\alpha}=(\alpha)$ consists only one component. That is, $\alpha$ is the unique number satisfying (1.14).

Finally, we show that there exists some $\sigma>0$ such that $z_{\ell}^{(\alpha)}(t)=o\left(e^{-\sigma t}\right)$ as $t \rightarrow \infty$ for $\ell=1,3,4$. We will first show that $N\left(\lambda,-\Delta_{\left.\mu_{2}\right|_{B_{n_{t}}, 3,2}}\right)$ is bounded.

Proposition 5.6. There exists a constant $C>0$ such that

$$
N\left(\lambda,-\Delta_{\left.\mu_{2}\right|_{B_{n_{t}}, 3,2}}\right) \leq C
$$

Proof. Let $A \subseteq B_{n_{t}, 3,2}=S_{e_{5} e_{3}^{n_{t}-2}}\left(B_{1,2}\right)=S_{e_{5} e_{3}^{n_{t}-1}}\left(\Omega_{1}\right)$. Then $S_{e_{5} e_{3}^{n_{t}-1}}^{-1}(A) \subseteq \Omega_{1}$. Hence

$$
\mu_{1}(A)=\left.p_{e_{5} e_{3}^{n_{t}-1}} \mu_{1}\right|_{\Omega_{1}} \circ S_{e_{5} e_{3}^{n_{t}-1}}^{-1} .
$$

Note that $S_{e_{5}} S_{e_{5} e_{3}^{n_{t}-1}}^{-1}(A) \subseteq B_{1,3}$ and for any $B \subseteq B_{1,3}$, we have $S_{e_{5}}^{-1}(B) \subseteq \Omega_{1}, \mu_{2}(B)=$ $\left.p_{e_{5}} \mu_{1}\right|_{\Omega_{1}} \circ S_{e_{5}}^{-1}$, and hence $\left.\mu_{1}\right|_{\Omega_{1}}=\left.p_{e_{5}}^{-1} \mu_{2}\right|_{B_{1,3}} \circ S_{e_{5}}$. Combining this with (5.9), we get

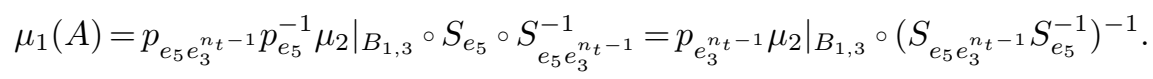

So

$$
\left.\mu_{1}\right|_{S_{e_{5} e_{3}^{n_{t}-2}}\left(B_{1,2}\right)}=\left.p_{e_{3}^{n_{t}-1}} \mu_{2}\right|_{B_{1,3}} \circ\left(S_{e_{5} e_{3}^{n_{t}-1}} S_{e_{5}}^{-1}\right)^{-1} \quad \text { on } S_{e_{5} e_{3}^{n_{t}-2}}\left(B_{1,2}\right) .
$$

It follows that

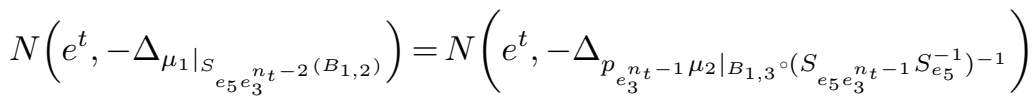

$$
\begin{aligned}
& =N\left(\left(p_{e_{3}} r\right)^{n_{t}-1} e^{t},-\Delta_{\left.\mu_{2}\right|_{B_{1,3}}}\right) \text {. }
\end{aligned}
$$

(4.11) implies that $\left(p_{e_{3}} r\right)^{n_{t}-1} e^{t}<\left(p_{e_{5}} \rho\right)^{-1} e^{t_{0}}$. Hence

$$
N\left(e^{t},-\Delta_{\left.\mu_{1}\right|_{S_{5} e_{3}^{n_{t}-2}\left(B_{1,2}\right)}}\right) \leq N\left(\left(p_{e_{5}} \rho\right)^{-1} e^{t_{0}},-\Delta_{\left.\mu_{2}\right|_{B_{1,3}}}\right):=C .
$$

This completes the proof.

Proposition 5.7. Let $\alpha$ be defined as in (1.14). Then there exists some $\sigma>0$ such that $z_{\ell}^{(\alpha)}(t)=o\left(e^{-\sigma t}\right)$ as $t \rightarrow \infty$ for $\ell=1,3,4$.

Proof. It follows from Proposition 5.6 that there exists some constant $C>0$ such that $z_{3}^{(\alpha)}(t) \leq\left(C+4 n_{t}-4\right) e^{-\alpha t}$. Moreover, since $z_{\ell}^{(\alpha)}(t) \leq 2 e^{-\alpha t}$ for $\ell=1,4$, 
it suffices to show that for any $0<\sigma<\alpha, n_{t} e^{-\alpha t}=o\left(e^{-\sigma t}\right)$ as $t \rightarrow \infty$. It follows from (4.11) that $t+\ln \left(p_{e_{5} e_{3}^{n_{t}-1}} \rho r^{n_{t}-1}\right)<t_{0}$, and hence $n_{t} \leq 1+\left(\ln \left(p_{e_{3}} r\right)\right)^{-1}\left(t_{0}-t-\right.$ $\left.\ln \left(p_{e_{5}} \rho\right)\right)$. Thus for any $0<\sigma<\alpha$,

$$
n_{t} e^{-\alpha t} / e^{-\sigma t} \leq\left(1+\left(\ln \left(p_{e_{3}} r\right)\right)^{-1}\left(t_{0}-t-\ln \left(p_{e_{5}} \rho\right)\right)\right) / e^{(\alpha-\sigma) t} \longrightarrow 0 \quad \text { as } t \longrightarrow \infty .
$$

This completes the proof.

Proof of Corollary 1.3. Apply Propositions 5.5 and 5.7, and Theorem 1.1.

Proof of Remark 1.4. (a) follows by a direct calculation.

(b) It is easy to see that $G=(V, E)$ defined as in (1.12) satisfies the generalized finite type condition (see [20, Definition 2.1]) with $\left\{\Omega_{1}, \Omega_{2}\right\}=\{(0,1),(0,1)\}$ being a generalized finite type condition family for $G$. Moreover, the weighted incidence matrix is

$$
A_{x}:=\left(\begin{array}{cccc}
r^{x} & 0 & \rho^{x} & r^{x} \\
\rho^{x} & r^{x} & 0 & 0 \\
r^{x} & 0 & \rho^{x} & r^{x} \\
0 & r^{x} & 0 & 0
\end{array}\right)
$$

Let $x_{0}$ be the unique real number such that the spectral radius of $A_{x_{0}}$ is equal to 1. It follows from [20, Theorem 1.1] that the Hausdorff dimension of the associated graph self-similar set is $d_{f}=x_{0}=0.710396 \ldots$. Hence $2 d_{f} /\left(1+d_{f}\right)=0.830680 \ldots$, and so $d_{s}<2 d_{f} /\left(1+d_{f}\right)$.

(c) It follows from $\left[12\right.$, Lemma 2.3] that $\left\langle\operatorname{supp}\left(\nu_{1}\right)\right\rangle$ equals the closed subgroup generated by $\operatorname{supp}\left(\mu_{11}^{(\alpha)}\right)$, the closure of $\operatorname{supp}\left(\mu_{13}^{(\alpha)}\right)+\operatorname{supp}\left(\mu_{31}^{(\alpha)}\right)$, and the closure of $\operatorname{supp}\left(\mu_{14}^{(\alpha)}\right)+\operatorname{supp}\left(\mu_{43}^{(\alpha)}\right)+\operatorname{supp}\left(\mu_{31}^{(\alpha)}\right)$. By (5.8), we have

$$
\begin{array}{ll}
\operatorname{supp}\left(\mu_{11}^{(\alpha)}\right)=\left\{-\ln \left(p_{e_{1}} \rho\right)\right\}, & \operatorname{supp}\left(\mu_{31}^{(\alpha)}\right)=\left\{-\ln \left(p_{e_{5} e_{3}^{k}} \rho r^{k}\right): k \geq 0\right\}, \\
\operatorname{supp}\left(\mu_{13}^{(\alpha)}\right)=\left\{-\ln \left(\left(p_{e_{1} e_{3}}+p_{e_{2} e_{5}}\right) p_{e_{5}}^{-1} r\right)\right\}, & \operatorname{supp}\left(\mu_{14}^{(\alpha)}\right)=\left\{-\ln \left(p_{e_{2}} r\right)\right), \\
\operatorname{supp}\left(\mu_{43}^{(\alpha)}\right)=\operatorname{supp}\left(\mu_{44}^{(\alpha)}\right)=\left\{\ln \left(p_{e_{4}} r\right)\right\} . &
\end{array}
$$

More precisely,

$$
\begin{aligned}
& \operatorname{supp}\left(\mu_{11}^{(\alpha)}\right)=\left\{-\ln \left(p_{e_{1}} \rho\right)\right\}=\{\ln (12)\} \\
& \begin{aligned}
\operatorname{supp}\left(\mu_{13}^{(\alpha)}\right)+\operatorname{supp}\left(\mu_{31}^{(\alpha)}\right)=\left\{-\ln \left(\left(p_{e_{1} e_{3}}+p_{e_{2} e_{5}}\right) p_{e_{3}^{k}} \rho r^{k+1}\right): k \geq 0\right\} \\
=\left\{\ln \left(6 \times 7^{k+1}\right): k \geq 0\right\}, \\
\operatorname{supp}\left(\mu_{14}^{(\alpha)}\right)+\operatorname{supp}\left(\mu_{43}^{(\alpha)}\right)+\operatorname{supp}\left(\mu_{31}^{(\alpha)}\right)=\left\{\ln \left(p_{e_{2} e_{3}^{k} e_{4} e_{5}} \rho r^{k+2}\right): k \geq 0\right\} \\
=\left\{\ln \left(12 \times 7^{k+2}\right): k \geq 0\right\} .
\end{aligned}
\end{aligned}
$$


Choose

$$
\begin{aligned}
& a:=\ln (6 \times 7) \in \operatorname{supp}\left(\mu_{13}^{(\alpha)}\right)+\operatorname{supp}\left(\mu_{31}^{(\alpha)}\right), \\
& b:=\ln \left(12 \times 7^{2}\right) \in \operatorname{supp}\left(\mu_{14}^{(\alpha)}\right)+\operatorname{supp}\left(\mu_{43}^{(\alpha)}\right)+\operatorname{supp}\left(\mu_{31}^{(\alpha)}\right) .
\end{aligned}
$$

Since $a / b \notin \mathbf{Q}$, we have $\left\langle\operatorname{supp}\left(\nu_{1}\right)\right\rangle=\mathbf{R}$ and hence $\nu_{1}$ is non-lattice. Combining this with Theorem 1.1(c), we see that there exists a non-negative constant $c_{\ell}$ such that

$$
\lim _{t \rightarrow \infty} e^{-\alpha t} N\left(e^{t},-\Delta_{\left.\mu_{i}\right|_{B_{1, \ell}}}\right)=c_{\ell}, \text { for } \ell \in \Gamma_{i}, \ell=1,3,4 \text {, and } i=1,2,
$$

which completes the proof.

\section{GIFSs that are not strongly connected}

In this section, we compute the spectral dimension of some graph-directed selfsimilar measures defined by the GIFSs $G=(V, E)$ which have overlaps and are not strongly connected.

\subsection{A GIFS that is not strongly connected and has a unique basic class}

It is easy to check that the GIFS in Example 1.7 is not strongly connected and has overlaps. In this subsection, we show that the associated measures satisfy (EFT). In Subsection 6.2 we show that this GIFS has a unique basic class, and compute the spectral dimension of the corresponding measures.

Proposition 6.1. Let $\mu=\mu_{1}+\mu_{2}$ be a graph-directed self-similar measure defined by a $G I F S G=(V, E)$ in Example 1.7 together with a probability matrix $\left(p_{e}\right)_{e \in E}$. Then $\mu$ satisfies $(E F T)$ with $\Omega=\left\{\Omega_{1}, \Omega_{2}\right\}=\{(0,1),(0,1)\}$ being an EFT-family; moreover, there exists a regular basic pair.

To prove Proposition 6.1, we first summarize some elementary properties.

Throughout this subsection, we let $\Omega_{1}=\Omega_{2}:=(0,1)$. To simplify notation we let

$$
\zeta_{k}:=1-r^{k}, \quad k \geq 0
$$

Define

$$
\begin{array}{ll}
B_{1,1}:=S_{e_{1}}\left(\Omega_{1}\right) \cup S_{e_{2}}\left(\Omega_{1}\right)=\left(0, r+\rho \zeta_{1}\right), & B_{1,2}:=S_{e_{3}}\left(\Omega_{1}\right)=\left(\zeta_{1}, 1\right), \\
B_{1,3}:=S_{e_{5}}\left(\Omega_{1}\right)=(0, \rho), & B_{1,4}:=S_{e_{4}}\left(\Omega_{2}\right)=\left(\zeta_{1}, 1\right) .
\end{array}
$$

We denote

$$
W(k)=\left\{e_{2}^{j} e_{1} e_{3}^{k-j}: j=0,1, \ldots, k\right\}, \quad k \geq 0
$$


We remark that for $k \geq 0$,

$$
p_{e_{1}} p_{e_{3}}^{k+1}+p_{e_{2}} w(k)=w(k+1) \quad \text { and } \quad w(k+1) \leq w(k) \leq p_{e_{1}},
$$

where $w(k)$ is defined as in (1.16). For any $k \geq 0, w(k)$ denotes the sum of probability weights of all multi-indices in $W(k)$. Using (2.3) and (2.4), we see that

$$
p_{e_{1}}+p_{e_{2}}+p_{e_{3}}=1, \quad p_{e_{4}}+p_{e_{5}}=1,
$$

and

$$
\mu_{1}=p_{e_{1}} \mu_{1} \circ S_{e_{1}}^{-1}+p_{e_{2}} \mu_{1} \circ S_{e_{2}}^{-1}+p_{e_{3}} \mu_{1} \circ S_{e_{3}}^{-1}, \mu_{2}=p_{e_{4}} \mu_{2} \circ S_{e_{4}}^{-1}+p_{e_{5}} \mu_{1} \circ S_{e_{5}}^{-1}
$$

Moreover, $\mu=\mu_{1}+\mu_{2}$.

Lemma $6.2(\mathrm{a})$ below implies that all multi-indices in $W(k)$ correspond to the same vertex.

Lemma 6.2. Let $\left\{S_{e_{i}}\right\}_{i=1}^{5}$ be as in (1.15). Then

(a) $S_{e_{1} e_{3}}\left(\Omega_{1}\right)=S_{e_{2} e_{1}}\left(\Omega_{1}\right)$. Moreover, for any $\boldsymbol{e}, \boldsymbol{e}^{\prime} \in W(k), S_{\boldsymbol{e}}=S_{\boldsymbol{e}^{\prime}}$

(b) for $k \geq 1$,

$$
\begin{aligned}
& S_{e_{2}^{k} e_{1}}\left(\Omega_{1}\right)=\left(\rho \zeta_{k}, \rho\right), \quad S_{e_{2}^{k}}\left(\Omega_{1}\right)=\left(\rho \zeta_{k}, r^{k}+\rho \zeta_{k}\right), \\
& S_{e_{2}^{k} e_{3}}\left(\Omega_{1}\right)=\left(r^{k} \zeta_{1}+\rho \zeta_{k}, r^{k}+\rho \zeta_{k}\right), \quad S_{e_{1}}\left(\Omega_{1}\right) \cap S_{e_{2}^{k}}\left(\Omega_{1}\right)=S_{e_{2}^{k} e_{1}}\left(\Omega_{1}\right) \text {. }
\end{aligned}
$$

Proof. (a) can be proved by a direct calculation and (b) can be proved by induction; we omit the details.

Part (d) of the following lemma explains the meaning of the factor $w(k)$.

Lemma 6.3. Assume the hypotheses of Proposition 6.1. Let $w(k)$ be defined as in (1.16). Then

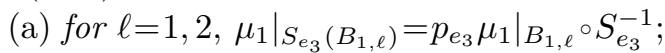

(b) for $\ell=3,4,\left.\mu_{2}\right|_{S_{e_{4}}\left(B_{1, \ell}\right)}=\left.p_{e_{4}} \mu_{2}\right|_{B_{1, \ell}} \circ S_{e_{4}}^{-1}$;

(c) for $\ell=1,2,\left.\mu_{2}\right|_{S_{e_{5}}\left(B_{1, \ell}\right)}=\left.p_{e_{5}} \mu_{1}\right|_{B_{1, \ell}} \circ S_{e_{5}}^{-1}$;

(d) for $k \geq 0,\left.\mu_{1}\right|_{S_{2}^{k} e_{1}}\left(B_{1,1}\right)=\left.w(k) \mu_{1}\right|_{B_{1,1}} \circ S_{e_{2}^{k} e_{1}}^{-1}$;

(e) for $k \geq 1,\left.\mu_{1}\right|_{S_{e_{2}^{k}}\left(B_{1,1}\right)}=\left.w(k-1) \mu_{1}\right|_{B_{1,2}} \circ S_{e_{2}^{k-1} e_{1}}^{-1}+\left.p_{e_{2}}^{k} \mu_{1}\right|_{B_{1,1}} \circ S_{e_{2}^{k}}^{-1}$;

(f) for $k \geq 1,\left.\mu_{1}\right|_{S_{e_{2}^{k}}\left(B_{1,2}\right)}=\left.p_{e_{2}}^{k} \mu_{1}\right|_{B_{1,2}} \circ S_{e_{2}^{k}}^{-1}$.

Proof. (a)-(c) follow from (6.6) along with the facts that $S_{e_{i}}\left(B_{1, \ell}\right) \subseteq S_{e_{i}}\left(\Omega_{1}\right)$ and $S_{e_{4}}\left(B_{1, \ell^{\prime}}\right) \subseteq S_{e_{4}}\left(\Omega_{2}\right)$, for $i=3,5, \ell=1,2$ and $\ell^{\prime}=3,4$.

(d)-(f) can be proved directly by induction, we only prove (d) as an example. It follows from (1.15) and (6.2) that $S_{e_{1}}\left(\Omega_{1}\right)=(0, \rho), S_{e_{2}}\left(\Omega_{2}\right)=\left(\rho \zeta_{1}, r+\right.$ $\left.\rho \zeta_{1}\right)$, and $S_{e_{1}}\left(B_{1,1}\right)=\left(0, \rho r+\rho^{2} \zeta_{1}\right)$. Since $\rho+2 r-\rho r \leq 1$, we have $\rho r+\rho^{2} \zeta_{1} \leq \rho \zeta_{1}$, 

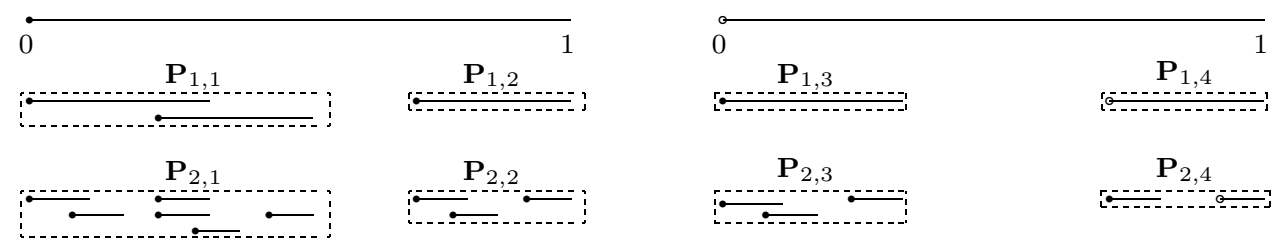

Figure 6. $\mu$-partitions $\mathbf{P}_{k, \ell}$ of $B_{1, \ell}$ for the GIFS in (1.15). The figure is drawn with $\rho=1 / 3$ and $r=2 / 7$.

and hence $S_{e_{1}}\left(B_{1,1}\right) \subseteq S_{e_{1}}\left(\Omega_{1}\right) \backslash S_{e_{2}}\left(\Omega_{2}\right)$. So for any $A \subseteq S_{e_{1}}\left(B_{1,1}\right)$, i.e., $S_{e_{1}}^{-1}(A) \subseteq$ $B_{1,1}$, we get $\mu_{1}(A)=\left.p_{e_{1}} \mu_{1}\right|_{B_{1,1}} \circ S_{e_{1}}^{-1}(A)$. Assume that the stated equality holds for $k=m$, i.e., $\left.\mu_{1}\right|_{S_{e_{2}^{m} e_{1}}\left(B_{1,1}\right)}=\left.w(m) \mu_{1}\right|_{B_{1,1}} \circ S_{e_{2}^{m} e_{1}}^{-1}$. For $k=m+1$, by Lemma 6.2 , we have $S_{e_{2}^{m+1} e_{1}}\left(B_{1,1}\right)=S_{e_{1} e_{3}^{m+1}}\left(B_{1,1}\right)$. Then $S_{e_{1}}^{-1}(A) \subseteq S_{e_{3}^{m+1}}\left(B_{1,1}\right)$ and $S_{e_{2}}^{-1}(A) \subseteq$ $S_{e_{2}^{m} e_{1}}\left(B_{1,1}\right)$ for any $A \subseteq S_{e_{2}^{m+1} e_{1}}\left(B_{1,1}\right)$. It follows that $\mu_{1}\left(S_{e_{1}}^{-1}(A)\right)=\left.p_{e_{3}}^{m+1} \mu_{1}\right|_{B_{1,1}}$ 。 $S_{e_{3}^{m+1}}^{-1}\left(S_{e_{1}}^{-1}(A)\right)$ and $\mu_{1}\left(S_{e_{2}}^{-1}(A)\right)=\left.w(m) \mu_{1}\right|_{B_{1,1}} \circ S_{e_{2}^{m} e_{1}}^{-1}\left(S_{e_{2}}^{-1}(A)\right)$. Thus,

$$
\begin{aligned}
\mu_{1}(A) & =p_{e_{1}} \mu_{1} \circ S_{e_{1}}^{-1}(A)+p_{e_{2}} \mu_{1} \circ S_{e_{2}}^{-1}(A) \\
& =\left.p_{e_{1}} p_{e_{3}}^{m+1} \mu_{1}\right|_{B_{1,1}} \circ S_{e_{3}^{m+1}}^{-1}\left(S_{e_{1}}^{-1}(A)\right)+\left.p_{e_{2}} w(m) \mu_{1}\right|_{B_{1,1}} \circ S_{e_{2}^{m} e_{1}}^{-1}\left(S_{e_{2}}^{-1}(A)\right) \\
& =\left.\left(p_{e_{1}} p_{e_{3}}^{m+1}+p_{e_{2}} w(m)\right) \mu_{1}\right|_{B_{1,1}} \circ S_{e_{2}^{m+1} e_{1}}^{-1}(A) \\
& =\left.w(m+1) \mu_{1}\right|_{B_{1,1}} \circ S_{e_{2}^{m+1} e_{1}}^{-1}(A) .
\end{aligned}
$$

The last equality follows from (6.4). This proves part (d).

Proof of Proposition 6.1. Note that $\left\{\Omega_{1}, \Omega_{2}\right\}$ is invariant under $G=(V, E)$. It is obvious that the elements of $\left\{B_{1, i}\right\}_{i=1}^{4}$ are measure disjoint. Let $\Gamma:=\{1,2,3,4\}$ and $\mathbf{B}:=\left\{B_{1, \ell}: \ell \in \Gamma\right\}$. Define $\mathbf{P}_{1, \ell}:=\left\{B_{1, \ell}\right\}$ for $\ell \in \Gamma$. If for some $k \geq 2$ and $\ell \in \Gamma, \mathbf{P}_{k, \ell}$ (see Figure 6) is a well-defined $\mu$-partition of $B_{1, \ell}$, then we let $\mathbf{P}_{k, \ell}^{1}$ and $\mathbf{P}_{k, \ell}^{2}$ be defined as in (2.6) with respect to $\mathbf{B}$.

For $\ell=1$, define $\mathbf{P}_{2,1}:=\left\{S_{e_{1}}\left(B_{1,1}\right), S_{e_{2}}\left(B_{1,1}\right), S_{e_{2}}\left(B_{1,2}\right)\right\}$ (see Figure 7$)$. By Lemma $6.3(\mathrm{~d}, \mathrm{e}, \mathrm{f})$, we have $\mathbf{P}_{2,1}^{1}=\left\{S_{e_{1}}\left(B_{1,1}\right), S_{e_{2}}\left(B_{1,2}\right)\right\}$ and $\mathbf{P}_{2,1}^{2}=\left\{S_{e_{2}}\left(B_{1,1}\right)\right\}$. Hence condition (1) of (EFT) holds for $\ell=1$. For $k \geq 3$, define

$$
\mathbf{P}_{k, 1}:=\mathbf{P}_{k-1,1} \cup\left\{S_{e_{2}^{k-2} e_{1}}\left(B_{1,1}\right), S_{e_{2}^{k-1}}\left(B_{1,1}\right), S_{e_{2}^{k-1}}\left(B_{1,2}\right)\right\} .
$$

By Lemma 6.3(d,e,f), we get $\mathbf{P}_{k, 1}^{1}=\mathbf{P}_{k-1,1}^{1} \cup\left\{S_{e_{2}^{k-2} e_{1}}\left(B_{1,1}\right), S_{e_{2}^{k-1}}\left(B_{1,2}\right)\right\}$ and $\mathbf{P}_{k, 1}^{2}=$ $\left\{S_{e_{2}^{k-1}}\left(B_{1,1}\right)\right\}$. Hence condition $(2)$ of (EFT) holds for $\ell=1$. Since the closure of $S_{e_{2}^{k}}\left(B_{1,1}\right)$ converges to a point as $k \rightarrow \infty$, we have $\lim _{k \rightarrow \infty} \mu\left(S_{e_{2}^{k}}\left(B_{1,1}\right)\right)=0$. Thus condition (3) of (EFT) holds. 


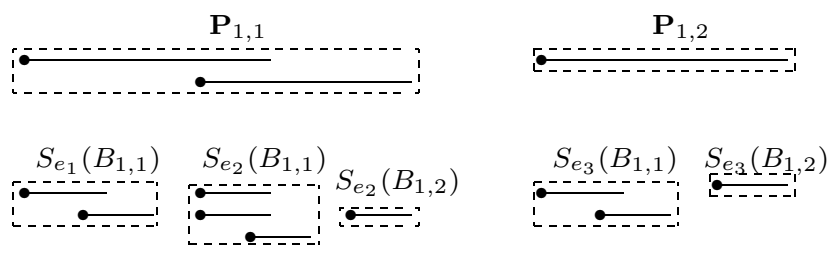

Figure 7. $\mu$-partitions $\mathbf{P}_{2,1}$ and $\mathbf{P}_{2,2}$.

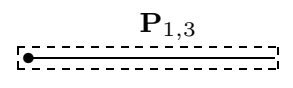

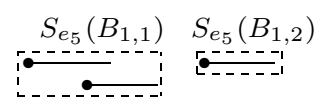

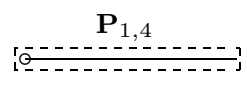

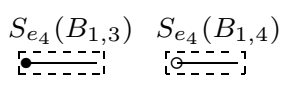

Figure 8. $\mu$-partitions $\mathbf{P}_{2,3}$ and $\mathbf{P}_{2,4}$.

For $\ell=2$, define $\mathbf{P}_{2,2}:=\left\{S_{e_{3}}\left(B_{1,1}\right), S_{e_{3}}\left(B_{1,2}\right)\right\}$ (see Figure 7 ). Note that $S_{e_{3}}\left(B_{1,1}\right), S_{e_{3}}\left(B_{1,2}\right)$ are measure disjoint, and $S_{e_{3}}\left(B_{1, i}\right) \subseteq B_{1,2}$ for $i=1,2$. Hence $\mathbf{P}_{2,2}$ is a refining $\mu$-partition of $B_{1,2}$. It follows from Lemma 6.3(a) that $\mathbf{P}_{2,2}^{1}=\mathbf{P}_{2,2}$ and $\mathbf{P}_{2,2}^{2}=\varnothing$. Thus condition (1) of (EFT) holds for $\ell=2$. For $k>2$, define $\mathbf{P}_{k, 2}:=$ $\mathbf{P}_{2,2}$. It follows that conditions (2) and (3) of (EFT) hold for $\ell=2$.

For $\ell=3$, define $\mathbf{P}_{2,3}:=\left\{S_{e_{5}}\left(B_{1,1}\right), S_{e_{5}}\left(B_{1,2}\right)\right\}$ (see Figure 8). Note that $S_{e_{5}}\left(B_{1,1}\right), S_{e_{5}}\left(B_{1,2}\right)$ are measure disjoint, and $S_{e_{5}}\left(B_{1, i}\right) \subseteq B_{1,3}$ for $i=1,2$. Hence $\mathbf{P}_{2,3}$ is a refining $\mu$-partition of $B_{1,3}$. It follows from Lemma $6.3(\mathrm{c})$ that $\mathbf{P}_{2,3}^{1}=\mathbf{P}_{2,3}$ and $\mathbf{P}_{2,3}^{2}=\varnothing$. Thus condition (1) of (EFT) holds for $\ell=3$. For $k>2$, define $\mathbf{P}_{k, 3}:=$ $\mathbf{P}_{2,3}$. It follows that conditions (2) and (3) of (EFT) hold for $\ell=3$.

For $\ell=4$, define $\mathbf{P}_{2,4}:=\left\{S_{e_{4}}\left(B_{1,3}\right), S_{e_{4}}\left(B_{1,4}\right)\right\}$ (see Figure 8). Note that $S_{e_{4}}\left(B_{1,3}\right), S_{e_{4}}\left(B_{1,4}\right)$ are measure disjoint, and $S_{e_{4}}\left(B_{1, i}\right) \subseteq B_{1,4}$ for $i=3,4$. Hence $\mathbf{P}_{2,4}$ is a refining $\mu$-partition of $B_{1,4}$. It follows from Lemma $6.3(\mathrm{~b})$ that $\mathbf{P}_{2,4}^{1}=\mathbf{P}_{2,4}$ and $\mathbf{P}_{2,4}^{2}=\varnothing$. Thus condition (1) of (EFT) holds for $\ell=4$. For $k>2$, define $\mathbf{P}_{k, 4}:=$ $\mathbf{P}_{2,4}$. It follows that conditions (2) and (3) of (EFT) hold for $\ell=4$.

\subsection{Spectral dimension of $\mu$ in Proposition 6.1}

In this subsection, we derive vector-valued renewal equations and compute the spectral dimension of the measure $\mu$ defined by the GIFS in Proposition 6.1. We also show that the GIFS has a unique basic class.

Let $\left\{S_{e_{i}}\right\}_{i=1}^{5}$ be a GIFS in (1.15), $\left(p_{e}\right)_{e \in E}$ be a probability matrix, and $\mu$ be the associated graph-directed self-similar measure. For $\ell \in \Gamma:=\{1,2,3,4\}$ and $k \geq 1$, 
let $B_{1, \ell}$ be defined as in (6.2), and $\mathbf{P}_{k, \ell}$ be as in the proof of Proposition 6.1. Then $\mu$ satisfies (EFT) with $\left\{\Omega_{1}, \Omega_{2}\right\}=\{(0,1),(0,1)\}$ being an EFT-family, and $(\mathbf{B}, \mathbf{P}):=\left(\left\{B_{1, \ell}\right\},\left\{\mathbf{P}_{k, \ell}\right\}_{k \geq 1}\right)_{\ell \in \Gamma}$ being a regular basic pair.

In the rest of this subsection, we use the notation in Section 4.1. For $\ell \in \Gamma$, $i=1,2$ and $k \geq 2$, let $\mathbf{P}_{k, \ell}^{i}$ be defined as in (2.6). For $i=1,2$, let $\Gamma_{i}$ and $\mathbf{B}_{i}$ be defined as in (4.1). Since $B_{1, \ell} \subseteq \Omega_{1}$ and $B_{1, \ell^{\prime}} \subseteq \Omega_{2}$ for $\ell=1,2, \ell^{\prime}=3,4$, we have $\Gamma_{1}=\{1,2\}, \Gamma_{2}=\{3,4\}, \mathbf{B}_{1}=\left\{B_{1,1}, B_{1,2}\right\}$ and $\mathbf{B}_{2}=\left\{B_{1,3}, B_{1,4}\right\}$. For $k \geq 2$, let $B_{k, 1,1}=$ $S_{e_{2}^{k-2} e_{1}}\left(B_{1,1}\right), B_{k, 1,2}=S_{e_{2}^{k-1}}\left(B_{1,1}\right), B_{k, 1,3}=S_{e_{2}^{k-1}}\left(B_{1,2}\right)$, and let

$$
\begin{array}{ll}
B_{2,2, p}=S_{e_{3}}\left(B_{1, p}\right), & B_{2,3, p}=S_{e_{5}}\left(B_{1, p}\right), \text { for } p=1,2, \\
B_{2,4,1}=S_{e_{4}}\left(B_{1,3}\right), & B_{2,4,2}=S_{e_{4}}\left(B_{1,4}\right) .
\end{array}
$$

Then

$$
\begin{array}{ll}
\mathbf{P}_{k, 1}^{1}=\left\{B_{k, 1,1}, B_{k, 1,3}\right\}, & \mathbf{P}_{k, 1}^{2}=\left\{B_{k, 1,2}\right\}, \text { for } k \geq 2 \\
\mathbf{P}_{2, \ell}^{1}=\left\{B_{2, \ell, 1}, B_{2, \ell, 2}\right\}, & \mathbf{P}_{2, \ell}^{2}=\varnothing, \text { for } \ell=2,3,4 .
\end{array}
$$

Hence $\Gamma_{1}^{\prime}=\{2\}, \Gamma_{1}^{*}=\{1\}, \Gamma_{2}^{\prime}=\{3,4\}, \Gamma_{2}^{*}=\varnothing, \varkappa_{2}=\varkappa_{3}=\varkappa_{4}=2, \varkappa_{1}=\infty$, and

$$
\begin{array}{ll}
G_{k, 1}^{1}=\{1,3\}, & G_{k, 1}^{2}=\{2\}, \text { for } k \geq 2, \\
G_{2, \ell}^{1}=\{1,2\}, & G_{2, \ell}^{2}=\varnothing \text { for } \ell=2,3,4 .
\end{array}
$$

For $\ell \in \Gamma$, let $J_{\ell}$ be defined as in (4.3). Then $J_{1}=J_{2}=J_{3}=\{1\}$ and $J_{4}=\{2\}$.

Proposition 6.4. Let $\xi(\cdot, \cdot, \cdot)$ and $c(\cdot, \cdot, \cdot)$ be defined as in Proposition 4.2. Then

(a) for $k \geq 2, \xi(k, 1,1)=w(k-2) \rho r^{k-2}, c(k, 1,1)=1, \xi(k, 1,3)=\left(p_{e_{2}} r\right)^{k-1}$, and $c(k, 1,3)=2$;

(b) for $p=1,2$,

$$
\begin{array}{lll}
\xi(2,2, p)=p_{e_{3}} r, & \xi(2,3, p)=p_{e_{5}} \rho, & \xi(2,4, p)=p_{e_{4}} r, \\
c(2,2, p)=p, & c(2,3, p)=p, & c(2,4, p)=p+2 .
\end{array}
$$

Proof. Using Lemma 6.3(a)-(f), we have for $k \geq 2$,

$$
B_{1,1} \simeq_{\mu, S_{e_{2}^{k-2} e_{1}}, w(k-2)} B_{k, 1,1}, \quad B_{1,2} \simeq_{\mu, S_{e_{2}^{k-1}, p_{e_{2}}^{k-1}}} B_{k, 1,3},
$$

and for $p=1,2$,

$$
B_{1, p} \simeq_{\mu, S_{e_{3}}, p_{e_{3}}} B_{2,2, p}, \quad B_{1, p} \simeq_{\mu, S_{e_{5}}, p_{e_{5}}} B_{2,3, p}, \quad B_{1, p+2} \simeq_{\mu, S_{e_{4}, p_{e_{4}}}} B_{2,4, p} .
$$

The results follow. 
It is obvious that $G$ has two strongly connected components, i.e., $\eta=2$. For $m=1,2$, let $\mathcal{S C}_{m}$ be defined as in (1.6). Then $\mathcal{S C}_{1}=\{1\}$ and $\mathcal{S C}_{2}=\{2\}$.

Using Proposition 6.4 and the discussions preceding it, we can express the vector-valued renewal equations (4.16) and (4.20) precisely as

$$
\begin{aligned}
& f_{1}(t)=\sum_{k=0}^{\infty}\left(w(k) \rho r^{k}\right)^{\alpha_{1}} f_{1}\left(t+\ln \left(w(k) \rho r^{k}\right)\right)+\sum_{k=1}^{\infty}\left(p_{e_{2}} r\right)^{k \alpha_{1}} f_{2}\left(t+\ln \left(p_{e_{2}} r\right)^{k}\right)+z_{1}^{\left(\alpha_{1}\right)}(t) \\
& f_{2}(t)=\left(p_{e_{3}} r\right)^{\alpha_{1}} \sum_{\ell=1}^{2} f_{\ell}\left(t+\ln \left(p_{e_{3}} r\right)\right)+z_{2}^{\left(\alpha_{1}\right)}(t) \\
& f_{3}(t)=\left(p_{e_{5}} \rho\right)^{\alpha_{2}} \sum_{\ell=1}^{2} f_{\ell}\left(t+\ln \left(p_{e_{5}} \rho\right)\right)+z_{3}^{\left(\alpha_{2}\right)}(t) \\
& f_{4}(t)=\left(p_{e_{4}} r\right)^{\alpha_{2}} \sum_{\ell=3}^{4} f_{\ell}\left(t+\ln \left(p_{e_{4}} r\right)\right)+z_{4}^{\left(\alpha_{2}\right)}(t)
\end{aligned}
$$

where

$$
\begin{aligned}
& z_{1}^{\left(\alpha_{1}\right)}(t)=e^{-\alpha_{1} t} N\left(\lambda,-\Delta_{\left.\mu_{1}\right|_{B_{n_{t}, 1,2}}}\right)+e^{-\alpha_{1} t} \varepsilon\left(n_{t}, 1\right) \\
& z_{2}^{\left(\alpha_{1}\right)}(t)=e^{-\alpha_{1} t} \varepsilon(2,2), \quad z_{\ell}^{\left(\alpha_{2}\right)}(t)=e^{-\alpha_{2} t} \varepsilon(2, \ell) \quad \text { for } \ell=3,4 .
\end{aligned}
$$

For $\ell^{\prime}, \ell \in \Gamma$ and $m=1,2$, let $\mu_{\ell \ell^{\prime}}^{\left(\alpha_{m}\right)}$ be the discrete measure defined as in (4.21), and $\boldsymbol{\alpha}=\left(\alpha_{1}, \alpha_{2}\right)$. Then

$$
\begin{aligned}
& \mu_{11}^{\left(\alpha_{1}\right)}\left(-\ln \left(w(k) \rho r^{k}\right)\right)=\left(w(k) \rho r^{k}\right)^{\alpha_{1}}, \quad \text { for } k \geq 0, \\
& \mu_{12}^{\left(\alpha_{1}\right)}\left(-\ln \left(p_{e_{2}} r\right)^{k}\right)=\left(p_{e_{2}} r\right)^{k \alpha_{1}}, \quad \text { for } k \geq 1, \\
& \mu_{21}^{\left(\alpha_{1}\right)}\left(-\ln \left(p_{e_{3}} r\right)\right)=\mu_{22}^{\left(\alpha_{1}\right)}\left(-\ln \left(p_{e_{3}} r\right)\right)=\left(p_{e_{3}} r\right)^{\alpha_{1}}, \\
& \mu_{31}^{\left(\alpha_{2}\right)}\left(-\ln \left(p_{e_{5}} \rho\right)\right)=\mu_{32}^{\left(\alpha_{2}\right)}\left(-\ln \left(p_{e_{5}} \rho\right)\right)=\left(p_{e_{5}} \rho\right)^{\alpha_{2}}, \\
& \mu_{43}^{\left(\alpha_{2}\right)}\left(-\ln \left(p_{e_{4}} r\right)\right)=\mu_{44}^{\left(\alpha_{2}\right)}\left(-\ln \left(p_{e_{4}} r\right)\right)=\left(p_{e_{4}} r\right)^{\alpha_{2}} .
\end{aligned}
$$

Also,

$$
\mathbf{M}(\boldsymbol{\alpha} ; \infty)=\left(\begin{array}{cccc}
\sum_{k=0}^{\infty}\left(w(k) \rho r^{k}\right)^{\alpha_{1}} & \left(p_{e_{2}} r\right)^{\alpha_{1}} /\left(1-\left(p_{e_{2}} r\right)^{\alpha_{1}}\right) & 0 & 0 \\
\left(p_{e_{3}} r\right)^{\alpha_{1}} & \left(p_{e_{3}} r\right)^{\alpha_{1}} & 0 & 0 \\
\left(p_{e_{5}} \rho\right)^{\alpha_{2}} & \left(p_{e_{5}} \rho\right)^{\alpha_{2}} & 0 & 0 \\
0 & 0 & \left(p_{e_{4}} r\right)^{\alpha_{2}}\left(p_{e_{4}} r\right)^{\alpha_{2}}
\end{array}\right)
$$

Remark 6.5. The GIFS in Proposition 6.1 has a unique basic class, namely, $\{1,2\}$, which thus has height 1 by definition. Moreover, $\{1,2\}=\mathcal{S}_{0}$, where we recall that $\mathcal{S}_{0}$ is the union of basic classes of height 1 . 
Proposition 6.6. For $\ell=1,2,3,4$ and $m=1,2$, let $F_{\ell}\left(\alpha_{m}\right)$ and $\tilde{\alpha}_{\ell}$ be defined as in (1.10). Then $\lim _{\alpha_{m} \rightarrow \infty} F_{\ell}\left(\alpha_{m}\right)=0$ and $\tilde{\alpha}_{\ell}=0$ for $\ell=1,2,3,4$ and $m=1,2$. Moreover, $\lim _{\alpha_{1} \rightarrow 0^{+}} F_{1}\left(\alpha_{1}\right)=\infty$, and $F_{\ell}(0)>1$ for $\ell=2,3,4$.

Proof. By the definition of $F_{\ell}\left(\alpha_{m}\right)$, we see that

$$
\begin{aligned}
& F_{1}\left(\alpha_{1}\right)=\sum_{k=0}^{\infty}\left(w(k) \rho r^{k}\right)^{\alpha_{1}}+\left(p_{e_{2}} r\right)^{\alpha_{1}} /\left(1-\left(p_{e_{2}} r\right)^{\alpha_{1}}\right), \quad F_{2}\left(\alpha_{1}\right)=2\left(p_{e_{3}} r\right)^{\alpha_{1}}, \\
& F_{3}\left(\alpha_{2}\right)=2\left(p_{e_{5}} \rho\right)^{\alpha_{2}}, \quad F_{4}\left(\alpha_{2}\right)=2\left(p_{e_{4}} r\right)^{\alpha_{2}} .
\end{aligned}
$$

It follows from (6.4) that $F_{1}\left(\alpha_{1}\right)$ is a strictly decreasing positive continuous function of $\alpha_{1}$, and $\lim _{\alpha_{1} \rightarrow \infty} F_{1}\left(\alpha_{1}\right)=0$. It is obvious that $\lim _{\alpha_{m} \rightarrow \infty} F_{\ell}\left(\alpha_{m}\right)=0$ for $\ell=2,3,4$ and $m=1,2$. For any $\alpha_{1}>0$, since $\sum_{k=0}^{\infty}\left(w(k) \rho r^{k}\right)^{\alpha_{1}}$ converges, we get $F_{1}\left(\alpha_{1}\right)<\infty$. It follows from $(1.10)$ that $\widetilde{\alpha}_{1}=0$ and $\lim _{\alpha_{1} \rightarrow 0^{+}} F_{1}\left(\alpha_{1}\right)=\infty$. It is obvious that $\widetilde{\alpha}_{\ell}=0$ and $F_{\ell}(0)=2$ for $\ell=2,3,4$.

Let $\left|I_{4}-\mathbf{M}(\boldsymbol{\alpha} ; \infty)\right|=0$, where $I_{4}$ is the $4 \times 4$ identity matrix. Then

$$
\left[1-\left(p_{e_{4}} r\right)^{\alpha_{2}}\right]\left[1-\sum_{i=2}^{3}\left(p_{e_{i}} r\right)^{\alpha_{1}}-\left(\prod_{i=2}^{3}\left(1-\left(p_{e_{i}} r\right)^{\alpha_{1}}\right)\right) \sum_{k=0}^{\infty}\left(w(k) \rho r^{k}\right)^{\alpha_{1}}\right]=0
$$

It follows from Proposition 6.6 that there exists a unique set of non-negative real numbers $\alpha_{1}, \alpha_{2}$ such that both factors in the above equation equal zero; in particular, the spectral radius of $\mathbf{M}(\boldsymbol{\alpha} ; \infty)$ is 1 , where $\boldsymbol{\alpha}=\left(\alpha_{1}, \alpha_{2}\right)$. Note that $\alpha_{2}=0$. For the rest of this subsection, we let $\alpha:=\max \left\{\alpha_{1}, \alpha_{2}\right\}=\alpha_{1}$.

Finally, we show that there exists some $\sigma>0$ such that $z_{\ell}^{(\alpha)}(t)=o\left(e^{-\sigma t}\right)$ as $t \rightarrow \infty$ for $\ell=1,2,3,4$. We will first show that $N\left(\lambda,-\Delta_{\left.\mu_{1}\right|_{B_{n_{t}, 1,2}}}\right)$ is bounded. The proof is the same as that of [19, Proposition 5.3].

Proposition 6.7. There exists $C>0$ such that $N\left(\lambda,-\Delta_{\left.\mu_{1}\right|_{B_{n_{t}, 1,2}}}\right) \leq C$.

Proof. Let $A \subseteq B_{n_{t}, 1,2}=S_{e_{2}^{n_{t}-1}}\left(B_{1,1}\right)$. Then $S_{e_{2}^{n_{t}-2}}^{-1}(A) \subseteq S_{e_{2}}\left(B_{1,1}\right)=S_{e_{2} e_{1}}\left(\Omega_{1}\right) \cup$ $S_{e_{2} e_{2}}\left(\Omega_{1}\right)=S_{e_{1} e_{3}}\left(\Omega_{1}\right) \cup S_{e_{2} e_{2}}\left(\Omega_{1}\right)$. Thus

$$
\begin{aligned}
\left.\mu_{1}\right|_{S_{e_{2}^{n_{t}-2}}^{-1}(A)} & =\left.p_{e_{1}} \mu_{1}\right|_{B_{1,2}} \circ S_{e_{1}}^{-1}\left(S_{e_{2}^{n_{t}-2}}^{-1}(A)\right)+\left.p_{e_{2}} \mu_{1}\right|_{B_{1,1}} \circ S_{e_{2}}^{-1}\left(S_{e_{2}^{n_{t}-2}}^{-1}(A)\right) \\
& =\left.p_{e_{1}} \mu_{1}\right|_{B_{1,2}} \circ S_{e_{2}^{n_{t}-2} e_{1}}^{-1}(A)+\left.p_{e_{2}} \mu_{1}\right|_{B_{1,1}} \circ S_{e_{2}^{n_{t}-1}}^{-1}(A) .
\end{aligned}
$$


Multiplying both sides of (6.8) by $w\left(n_{t}-2\right) p_{e_{1}}^{-1}$, using (1.16) and Lemma 6.3(e), we have

$$
\begin{aligned}
& w\left(n_{t}-2\right) p_{e_{1}}^{-1} \mu_{1}\left(S_{e_{2}^{n_{t}-2}}^{-1}(A)\right) \\
= & \left.w\left(n_{t}-2\right) \mu_{1}\right|_{B_{1,2}} \circ S_{e_{2}^{n_{t}-2} e_{1}}^{-1}(A)+\left.w\left(n_{t}-2\right) p_{e_{2}} p_{e_{1}}^{-1} \mu_{1}\right|_{B_{1,1}} \circ S_{e_{2}^{n_{t}-1}}^{-1}(A) \\
\geq & \left.w\left(n_{t}-2\right) \mu_{1}\right|_{B_{1,2}} \circ S_{e_{2}^{n_{t}-2} e_{1}}^{-1}(A)+\left.p_{e_{2}}^{n_{t}-1} \mu_{1}\right|_{B_{1,1}} \circ S_{e_{2}^{n_{t}-1}}^{-1}(A) \\
= & \mu_{1}(A) .
\end{aligned}
$$

Thus $\left.\mu_{1}\right|_{S_{e_{2}}^{n_{t}-1}}\left(B_{1,1}\right) \leq w\left(n_{t}-2\right) p_{e_{1}}^{-1} \mu_{1} \circ S_{e_{2}^{n_{t}-2}}^{-1}$ on $S_{e_{2}^{n_{t}-1}}\left(B_{1,1}\right)$. Combining this with Proposition 3.4, we have

$$
N\left(e^{t},-\Delta_{\left.\mu_{1}\right|_{e_{2}^{n_{t}-1}\left(B_{1,1}\right)}}\right) \leq N\left(w\left(n_{t}-2\right) p_{e_{1}}^{-1} r^{n_{t}-2} e^{t},-\Delta_{\left.\mu_{1}\right|_{S_{e_{2}}\left(B_{1,1}\right)}}\right) .
$$

It follows from (4.19) that $t+\ln \left(w\left(n_{t}-1\right) \rho r^{n_{t}-1}\right)<t_{0}$. Hence we have

$$
w\left(n_{t}-2\right) \rho r^{n_{t}-1} e^{t} \leq w\left(n_{t}-1\right) \rho r^{n_{t}-1} e^{t}<e^{t_{0}},
$$

and thus $w\left(n_{t}-2\right) p_{e_{1}}^{-1} r^{n_{t}-2} e^{t} \leq\left(\rho r p_{e_{1}}\right)^{-1} e^{t_{0}}$. Combining this with (6.9), we get

$$
N\left(e^{t},-\Delta_{\left.\mu_{1}\right|_{S_{2}^{n_{t}-1}\left(B_{1,1}\right)}}\right) \leq N\left(\left(\rho r p_{e_{1}}\right)^{-1} e^{t_{0}},-\Delta_{\left.\mu_{1}\right|_{S_{e_{2}}\left(B_{1,1}\right)}}\right):=C .
$$

This completes the proof.

Proposition 6.8. There exists some $\sigma>0$ such that $z_{\ell}^{(\alpha)}(t)=o\left(e^{-\sigma t}\right)$ as $t \rightarrow \infty$ for $\ell=1,2,3,4$.

Proof. It follows from Proposition 6.7 that there exists some constant $C>0$ such that $z_{1}^{(\alpha)}(t) \leq\left(C+4 n_{t}-4\right) e^{-\alpha t}$. Moreover, since $z_{\ell}^{(\alpha)}(t) \leq 2 e^{-\alpha t}$ for $\ell=2,3,4$, it suffices to show that for any $0<\sigma<\alpha, n_{t} e^{-\alpha t}=o\left(e^{-\sigma t}\right)$ as $t \rightarrow \infty$. It follows from (4.19) that $t+\ln \left(p_{e_{2}} r\right)^{n_{t}}<t_{0}$, and hence $n_{t} \leq\left(\ln \left(p_{e_{2}} r\right)\right)^{-1}\left(t_{0}-t\right)$. Consequently for any $0<\sigma<\alpha$,

$$
n_{t} e^{-\alpha t} / e^{-\sigma t} \leq\left(\ln \left(p_{e_{2}} r\right)\right)^{-1} \cdot\left(t_{0}-t\right) / e^{(\alpha-\sigma) t} \longrightarrow 0 \quad \text { as } t \longrightarrow \infty .
$$

This completes the proof.

Proof of Corollary 1.8. Apply Propositions 6.6 and 6.8, and Theorem 1.6.

Proof of Remark 1.9. (a) follows by a direct calculation.

(b) It is obvious that $G=(V, E)$ defined as in (1.15) satisfies the generalized finite type condition with $\left\{\Omega_{1}, \Omega_{2}\right\}=\{(0,1),(0,1)\}$ being a generalized finite type 
condition family for $G$. Moreover, the weighted incidence matrix is

$$
A_{x}:=\left(\begin{array}{cccc}
r^{x} & 0 & \rho^{x} & r^{x} \\
\rho^{x} & r^{x} & 0 & 0 \\
0 & 0 & \rho^{x} & r^{x} \\
r^{x} & 0 & \rho^{x} & r^{x}
\end{array}\right) .
$$

Using [20, Theorem 1.1], we get $d_{f}=0.797012 \ldots$ Hence $2 d_{f} /\left(1+d_{f}\right)=0.887041 \ldots$, and thus $d_{s}<2 d_{f} /\left(1+d_{f}\right)$.

(c) It follows from [12, Lemma 2.3] that $\left\langle\operatorname{supp}\left(\nu_{1}\right)\right\rangle$ equals the closed group generated by $\operatorname{supp}\left(\mu_{11}^{(\alpha)}\right)$ and the closure of $\operatorname{supp}\left(\mu_{12}^{(\alpha)}\right)+\operatorname{supp}\left(\mu_{21}^{(\alpha)}\right)$, while $\left\langle\operatorname{supp}\left(\nu_{2}\right)\right\rangle$ equals the closed group generated by $\operatorname{supp}\left(\mu_{22}^{(\alpha)}\right)$ and the closure of $\operatorname{supp}\left(\mu_{21}^{(\alpha)}\right)+$ $\operatorname{supp}\left(\mu_{12}^{(\alpha)}\right)$. Using (6.7) with $\alpha_{1}=\alpha$, we have

$$
\begin{aligned}
& \operatorname{supp}\left(\mu_{11}^{(\alpha)}\right)=\left\{-\ln \left(w(k) \rho r^{k}\right): k \geq 0\right\} \\
& \operatorname{supp}\left(\mu_{12}^{(\alpha)}\right)=\left\{-\ln \left(p_{e_{2}} r\right)^{k}: k \geq 1\right\} \\
& \operatorname{supp}\left(\mu_{21}^{(\alpha)}\right)=\operatorname{supp}\left(\mu_{22}^{(\alpha)}\right)=\left\{-\ln \left(p_{e_{3}} r\right)\right\} .
\end{aligned}
$$

Hence

$$
\begin{aligned}
& \operatorname{supp}\left(\mu_{11}^{(\alpha)}\right)=\left\{-\ln \left(w(k) \rho r^{k}\right): k \geq 0\right\}=\left\{\ln \left(9 /(k+1) \cdot(21 / 2)^{k}\right): k \geq 0\right\}, \\
& \operatorname{supp}\left(\mu_{12}^{(\alpha)}\right)+\operatorname{supp}\left(\mu_{21}^{(\alpha)}\right)=\left\{-\ln \left(p_{e_{2}^{k} e_{3}} r^{k+1}\right): k \geq 1\right\}=\left\{\ln \left((21 / 2)^{k+1}\right): k \geq 1\right\}, \\
& \operatorname{supp}\left(\mu_{22}^{(\alpha)}\right)=\left\{-\ln \left(p_{e_{3}} r\right)\right\}=\{\ln (21 / 2)\} .
\end{aligned}
$$

It is easy to see that $\left\langle\operatorname{supp}\left(\nu_{2}\right)\right\rangle$ can be generated by $\ln (21 / 2)$, and so $\nu_{2}$ is lattice. Combining this with Theorem 1.6(c), we see that there exists a periodic function $q_{2}(t)$ such that

$$
\lim _{t \rightarrow \infty}\left(e^{-\alpha t} N\left(e^{t},-\Delta_{\left.\mu_{1}\right|_{B_{1,2}}}\right)-q_{2}(t)\right)=0 .
$$

Taking $a:=\ln (189 / 2) \in \operatorname{supp}\left(\mu_{11}^{(\alpha)}\right)$ and $b:=\ln \left(\left(21^{2}\right) / 4\right) \in \operatorname{supp}\left(\mu_{12}^{(\alpha)}\right)+\operatorname{supp}\left(\mu_{21}^{(\alpha)}\right)$, we have $a / b \notin \mathbf{Q}$. This leads to that $\left\langle\operatorname{supp}\left(\nu_{1}\right)\right\rangle=\mathbf{R}$, and so $\nu_{2}$ is non-lattice. Thus Theorem 1.6(c) implies that there exists a non-negative constant $q_{1}$ such that $\lim _{t \rightarrow \infty} e^{-\alpha t} N\left(e^{t},-\Delta_{\left.\mu_{1}\right|_{B_{1}, 1}}\right)=q_{1}$.

\subsection{A GIFS that is not strongly connected and has basic classes of height greater than 1}

The GIFS in Example 1.10 is not strongly connected and has overlaps. It differs from the one in Example 1.7 in that some of its basic classes have height greater than 1 . 
0

$$
\Omega_{1}
$$
10

$\Omega_{2}$

$\frac{S_{e_{1}}\left(\Omega_{1}\right)}{-} S_{e_{2}}\left(\Omega_{1}\right) \quad \quad S_{e_{3}}\left(\Omega_{1}\right)$

$\frac{S_{e_{4}}\left(\Omega_{1}\right)}{-} S_{e_{5}}\left(\Omega_{2}\right)$

$S_{e_{6}}\left(\Omega_{2}\right)$

0

$S_{e_{7}\left(\Omega_{3}\right)} S_{e_{8}}\left(\Omega_{3}\right) \quad S_{e_{9}}\left(\Omega_{3}\right)$

0

$S_{e_{13}\left(\Omega_{3}\right)} S_{e_{14}}\left(\Omega_{5}\right) \quad \quad \quad S_{e_{15}}\left(\Omega_{5}\right)$
10

\begin{tabular}{ll}
\hline & $\Omega_{4}$ \\
\hline$S_{e_{10}}\left(\Omega_{3}\right)$ & \\
\hline$-S_{e_{11}}\left(\Omega_{4}\right)$ & $S_{e_{12}}\left(\Omega_{4}\right)$
\end{tabular}

$\Omega_{6}$

$S_{e_{16}}\left(\Omega_{1}\right)$

1

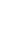

Figure 9. First iteration of the GIFS defined in $(1.18)$, where $\Omega_{i}=(0,1)$ for $i=1, \ldots, 6$. The figure is drawn with $\rho=1 / 3$ and $r=2 / 7$.

Proposition 6.9. Let $\mu=\sum_{i=1}^{6} \mu_{i}$ be a graph-directed self-similar measure defined by a GIFS $G=(V, E)$ in Example 1.10 together with a probability matrix $\left(p_{e}\right)_{e \in E}$. Then $\mu$ satisfies $(E F T)$ with

$$
\Omega=\left\{\Omega_{1}, \Omega_{2}, \Omega_{3}, \Omega_{4}, \Omega_{5}, \Omega_{6}\right\}=\{(0,1),(0,1),(0,1),(0,1),(0,1),(0,1)\}
$$

being an EFT-family and there exists a regular basic pair. Moreover, the GIFS has one basic class of height 2, and one of height 3 (see Figure 9).

Proof. As in Proposition 6.1, all the corresponding graph-directed self-similar measures $\mu$ satisfy (EFT); the proof is similar.

Let $\Gamma:=\{1,2, \ldots, 12\}$. There are five basic classes:

$$
\{1,2\}, \quad\{3,4\}, \quad\{5,6\}, \quad\{7,8\}, \quad\{9,10\} .
$$

Moreover, $1,2 \in \mathcal{S}_{1}, 5,6 \in \mathcal{S}_{2}$ and $3,4,7,8,9,10,11,12 \notin \mathcal{S}$, where $\mathcal{S}_{m}$ is the union of basic classes of height $m+1$ for $m \geq 0$ and $\mathcal{S}=\bigcup_{m \geq 0} \mathcal{S}_{m}$.

Proof of Corollary 1.11 and Remark 1.12. The computations of the spectral dimension of $\mu$ and the Hausdorff dimension of the associated graph self-similar set are similar to those in Proposition 6.1; we omit the details.

\section{A. Vector-valued renewal theorem}

For convenience, we state the multidimensional renewal theorem by Hambly and Nyberg [6], which is an extension of the results of Lau et al. [12]. This theorem is used in the proofs of Theorems 1.1 and 1.6. 
We introduce some terminology and refer the reader to [6] for any unexplained terms. Let $\mathbf{M}=\left[m_{i j}\right]_{n \times n}$ be a matrix of Radon measures on $\mathbf{R}_{+}$. We write $\mathbf{F}$ for the matrix of distribution functions of $\mathbf{M}$, that is $F_{i j}(t)=\int_{0}^{t} m_{i j} d s$ and write $F_{i j}(t, t+h]=F_{i j}(t+h)-F_{i j}(t)$. The indices of the matrix will be referred to as states. There is a directed edge between states $i$ and $j$ if the measure $m_{i j}$ is non-zero.

We follow [6] and define the measure

$$
\nu_{i}=m_{i i}+\mathrm{m}_{i \hat{i}} * \sum_{k=0}^{\infty}\left(\mathbf{M}_{i i}\right)^{* k} * \mathrm{~m}_{i \hat{i}}, \quad i=1, \ldots, n .
$$

Theorem A.1. (Hambly and Nyberg [6]) Assume that $\mathbf{F}(t)$ is a matrix of measures in which $\mathbf{F}(\infty)$ has maximum eigenvalue 1 , with $F_{i j}\left(0_{-}\right)=0, \int_{0}^{\infty} t d F_{i j}(t)<$ $\infty$ for all $i, j$ and for each $j$ there is at least one $i$ such that $F_{i j}(0)<F_{i j}(\infty)$. Let $\mathbf{z}$ be a vector with components that are directly Riemann integrable functions on $\mathbf{R}_{+}$ with $z_{i} \neq 0$ for all $i \in \mathcal{S}_{0}$. If $\mathbf{f}$ is continuous and satisfies the renewal equation

$$
\mathbf{f}(t)=\mathbf{f} * \mathbf{F}(t)+\mathbf{z}(t),
$$

then $\mathbf{f}=\mathbf{Z} * \sum_{k=0}^{\infty} \mathbf{F}^{* k}$ and the components $f_{i}$ satisfy

(1) if $i \in \mathcal{S}_{0}$, then

$$
\lim _{t \rightarrow \infty}\left(f_{i}(t)-q_{i}(t)\right)=0,
$$

where $q_{i}$ is either periodic or constant depending on whether $\nu_{i}$ is lattice or not;

(2) if $i \in \mathcal{S}_{m}$ for $m>0$, then

$$
\lim _{t \rightarrow \infty} t^{-m} f_{i}(t)=c_{i} \quad \text { for some constant } c_{i} \geq 0
$$

(3) if $i \notin \mathcal{S}=\bigcup_{m \geq 0} \mathcal{S}_{m}$ and there is no path from $\mathcal{S}$ to $i$, then

$$
\lim _{t \rightarrow \infty} f_{i}(t)=0
$$

(4) if $i \notin S$ and there is a path from $\mathcal{S}_{0}$ to $i$, but no path from $\mathcal{S}_{\ell}$ for any $\ell>0$, then

$$
\lim _{t \rightarrow \infty}\left(f_{i}(t)-\tilde{q}_{i}(t)\right)=0
$$

for some $\tilde{q}_{i}$ which is either constant or periodic;

(5) if $i \notin \mathcal{S}$ and there is a path from $\mathcal{S}_{m}$ to $i$, but no path from $\mathcal{S}_{\ell}$ for any $\ell>m>0$, then

$$
\lim _{t \rightarrow \infty} t^{-m} f_{i}(t)=\tilde{c}_{i} \quad \text { for some constant } \tilde{c}_{i} \geq 0 .
$$

If $i \in C_{k}$, an equivalence class in $\mathcal{S}_{m}$, then if $z_{j} \neq 0$ for at least one $j \in C_{k}$, we have $c_{i}, \tilde{c}_{i}>0$. 
Corollary A.2. (Hambly and Nyberg [6]) Let $t^{*}>0$, let $\mathbf{r}(t)$ be a vector whose components are measurable functions on $\mathbf{R}$ with $r_{i}(t)=0$, for all $i$ and $t<t^{*}$, and let $\mathbf{z}(t)$ be a non-negative directly Riemann integrable function with $z_{i}(t)=0$, for all $i$ and $t<t^{*}$. Assume that $\mathbf{r}$ satisfies the renewal equation

$$
\mathbf{r}(t)=\mathbf{z}(t)+(\mathbf{r} * \mathbf{F})(t), \quad t \in \mathbf{R} .
$$

Then the conclusions of Theorem A.1 hold.

Acknowledgements. The authors are very grateful to the anonymous referee for some valuable comments and suggestions.

\section{References}

1. Alonso-Ruiz, P. and Freiberg, U. R., Weyl asymptotics for Hanoi attractors, Forum Math. 29 (2017), 1003-1021.

2. Croydon, D. and Hambly, B., Self-similarity and spectral asymptotics for the continuum random tree, Stochastic Process. Appl. 118 (2008), 730-754.

3. Freiberg, U., Spectral asymptotics of generalized measure geometric Laplacians on Cantor like sets, Forum Math. 17 (2005), 87-104.

4. Fujita, T., A fractional dimension, self-similarity and a generalized diffusion operator, in Probabilistic methods in mathematical physics, Katata/Kyoto, 1985, pp. 8390, Academic Press, Boston, MA, 1987.

5. Hambly, B. M., On the asymptotics of the eigenvalue counting function for random recursive Sierpinski gaskets, Probab. Theory Related Fields 117 (2000), 221247.

6. Hambly, B. M. and Nyberg, S. O. G., Finitely ramified graph-directed fractals, spectral asymptotics and the multidimensional renewal theorem, Proc. Edinb. Math. Soc. 46 (2003), 1-34.

7. Hu, J., LAU, K.-S. and NGAI, S.-M., Laplace operators related to self-similar measures on $\mathbf{R}^{d}$, J. Funct. Anal. 239 (2006), 542-565.

8. Hutchinson, J. E., Fractals and self-similarity, Indiana Univ. Math. J. 30 (1981), $713-747$.

9. Kajino, N., Spectral asymptotics for Laplacians on self-similar sets, J. Funct. Anal. 258 (2010), 1310-1360.

10. Kajino, N., Log-periodic asymptotic expansion of the spectral partition function for self-similar sets, Comm. Math. Phys. 328 (2014), 1341-1370.

11. Kigami, J. and LAPIDUs, M. L., Weyl's problem for the spectral distribution of Laplacians on p.c.f. self-similar fractals, Comm. Math. Phys. 158 (1993), 93-125.

12. Lau, K.-S., WANG, J. and ChU, C.-H., Vector-valued Choquet-Deny theorem, renewal equation and self-similar measures, Studia Math. 117 (1995), 1-28.

13. Mauldin, R. D. and Williams, S. C., Hausdorff dimension in graph directed constructions, Trans. Amer. Math. Soc. 309 (1988), 811-829.

14. MaZ'JA, V. G., Sobolev spaces, Springer, Berlin, 1985. 
15. MCKeAn, H. P. and RAY, D. B., Spectral distribution of a differential operator, Duke Math. J. 29 (1962), 281-292.

16. Naimark, K. and Solomyak, M., The eigenvalue behaviour for the boundary value problems related to self-similar measures on $\mathbf{R}^{d}$, Math. Res. Lett. 2 (1995), 279-298.

17. NGAI, S.-M., Spectral asymptotics of Laplacians associated with one-dimensional iterated function systems with overlaps, Canad. J. Math. 63 (2011), 648-688.

18. NGai, S.-M. and TAng, W., Eigenvalue asymptotics and Bohr's formula for fractal Schrödinger operators, Pacific J. Math. 300 (2019), 83-119.

19. NGai, S.-M., TANG, W. and XIE, Y., Spectral asymptotics of one-dimensional fractal Laplacians in the absence of second-order identities, Discrete Contin. Dyn. Syst. 38 (2018), 1849-1887.

20. Ngai, S.-M., WAng, F. and Dong, X., Graph-directed iterated function systems satisfying the generalized finite type condition, Nonlinearity 23 (2010), 23332350.

21. Solomyak, M. and Verbitsky, E., On a spectral problem related to self-similar measures, Bull. London Math. Soc. 27 (1995), 242-248.

22. Strichartz, R. S., Taylor, A. and Zhang, T., Densities of self-similar measures on the line, Experiment. Math. 4 (1995), 101-128.

23. Wang, J. L., The open set condition for graph directed self-similar sets, Random Comput. Dynam. 5 (1997), 283-305.

24. Weyl, H., Das asymptotische Verteilungsgesetz der Eigenwerte linearer partieller Differentialgleichungen (mit einer Anwendung auf die Theorie der Hohlraumstrahlung), Math. Ann. 71 (1912), 441-479.

Sze-Man Ngai

Key Laboratory of High Performance

Computing and Stochastic Information

Processing (HPCSIP)

(Ministry of Education of China)

College of Mathematics and Statistics

Hunan Normal University

Changsha, Hunan CN-410081

P. R. China

and

Department of Mathematical Sciences

Georgia Southern University

Statesboro, GA 30460-8093

U.S.A.

smngai@georgiasouthern.edu

Received October 19, 2018

in revised form May 20, 2020
Yuanyuan Xie

School of Mathematics

Renmin University of China

Beijing, CN-100872

P. R. China

yyxiemath@163.com 